\title{
Imputação de Dados Categorizados Usando o Modelo Multinomial
}

\author{
Mamerto Granja Garcia
}

Dissertação apresentada ao

Instituto de Matemática e Estatística

da Universidade de São Paulo

para obtenção do título de

Mestre em Estatística

Área de Concentração: Estatística

Orientadora: Profa. Dra. Lúcia Pereira Barroso

São Paulo, Outubro de 2003 


\section{Imputação de Dados Categorizados Usando o Modelo Multinomial}

Este exemplar corresponde à redação final da dissertação devidamente corrigida e defendida por Mamerto Granja Garcia

e aprovada pela comissão julgadora.

São Paulo, Outubro de 2003.

Banca examinadora:

- Profa. Dra. Lúcia Pereira Barroso (orientadora) - IME/USP

- Prof. Dr. Júlio da Motta Singer - IME/USP

- Prof. Dr. Enrico Antonio Colosimo- UFMG 
Aos meus pais

Mamerto Granja Garcia e Manolvina Cunha,

pela dedicação e estímulo.

À minha esposa Lucilene

companheira compreensiva e incentivadora.

Aos meus filhos, Gustavo e Guilherme,

pelos constantes momentos felizes. 


\section{Agradecimentos}

Agradeço a Deus, por ter permitido que eu chegasse até aqui.

Quero agradecer à confiança em mim depositada por Lúcia Pereira Barroso, minha orientadora, demonstrando grande paciência e cordialidade.

Agradeço a Vladimir Stempliuk por ter permitido utilizar os dados da sua pesquisa relativa ao consumo de drogas entre os alunos da USP. Esses dados mostraramse de vital importância, em função de suas características específicas, para o desenvolvimento de boa parte deste trabalho.

Agradeço a todos aqueles que, direta ou indiretamente, colaboraram com este trabalho, principalmente à minha sogra e ao meu sogro, Maria Josete e José Pedro, pela ajuda com meus filhos, viabilizando tempo livre para realização deste trabalho. 


\section{Resumo}

Estamos interessados em mostrar como aproveitar observações incompletas, muitas vezes encontradas em estudos envolvendo dados categorizados em tabelas de contingência. Nesse sentido, propomos o ajuste de um modelo de censura aos dados observados, incluindo aqueles incompletos. O modelo poderá ser ignorável ou nãoignorável, permitindo-nos fazer uso da amostra por completo, por meio da imputação de dados.

Neste trabalho, ilustramos o ajuste do modelo de censura ignorável para tabelas de contingência com dados esparsos a uma pesquisa de consumo de drogas na Universidade de São Paulo. O modelo de censura não-ignorável também é descrito e é aplicado a dados de pesquisas eleitorais da eleição para Presidente do Brasil em 2002, segundo turno. 


\section{Sumário}

1 Introdução 2

2 Apresentação do Problema 4

2.1 Dados Observados . . . . . . . . . . . . . . . . . 4

2.2 Mecanismos de Censura . . . . . . . . . . . . . . . . 6

3 Imputação de Dados Categorizados sob o Modelo Probabilístico $\begin{array}{lr}\text { Multinomial } & 9\end{array}$

3.1 Mecanismo de Não-Resposta Ignorável . . . . . . . . . . . . . . . 9

3.1 .1 Estimação dos Parâmetros . . . . . . . . . . . . . . . 12

3.1 .2 Ajuste de Modelos Estruturais . . . . . . . . . . . . . . . . 14

3.1 .3 Aplicação . . . . . . . . . . . . . . . . . . 15

3.2 Mecanismo de Não-Resposta Não-Ignorável . . . . . . . . . . . . . . . 24

3.2 .1 O Modelo Log-linear . . . . . . . . . . . . . . . . . . . 25

3.2 .2 Estimação dos Parâmetros . . . . . . . . . . . . . . . 27

3.2 .3 Aplicação . . . . . . . . . . . . . . . . . . 32

$\begin{array}{llr}4 \text { Conclusões } & 39\end{array}$

A Estimação dos Parâmetros $\theta$ e $\lambda$. Modelo de Censura Ignorável. 41

B Variâncias de $\hat{\theta}$ e $\hat{\lambda}$. Modelo de Censura Ignorável. $\quad 49$

C Equações de Estimação para a Aplicação 2 
D Tópicos Utilizados $\quad 59$

D.1 Algoritmos . . . . . . . . . . . . . . . . . . . . . . . . 59

D.1.1 Algoritmo de Newton-Raphson . . . . . . . . . . . . 59

D.1.2 Algoritmo EM . . . . . . . . . . . . . . . . . 60

D.1.3 Algoritmo EM Generalizado . . . . . . . . . . . . . . . . . 62

D.1.4 Algoritmo IPF . . . . . . . . . . . . . . . . . 62

D.2 Ajuste de Modelos . . . . . . . . . . . . . . . . . . . . 64

D.3 Dados Esparsos . . . . . . . . . . . . . . . . . . . . . . . . . . 65

$\begin{array}{ll}\text { Referências Bibliográficas } & 72\end{array}$ 


\section{Lista de Figuras}

3.1 Gráfico indicando a variação do p-valor simulado em função da variação de $\alpha . \ldots \ldots \ldots$. . . . . . . . . . . . . . . . . . . . . . . . . 21

3.2 Gráficos representando as distribuições dos valores dos testes simulados de Pearson e RV para $\alpha$ igual a 0,01 e 0,32 juntamente com a distribuição $\chi^{2}$ com 7 graus de liberdade. . . . . . . . . . . . . . . . 22

3.3 Gráficos representando as distribuições dos valores dos testes simulados de Pearson e RV para $\alpha$ igual a 0,75 e 1,50 juntamente com a distribuição $\chi^{2}$ com 7 graus de liberdade. . . . . . . . . . . . . 23

3.4 Estimativa de $m_{\mathbf{i} j k}$, eleições 2002 , em função da escolha de $\Delta . \quad \ldots \quad 35$

3.5 Evolução das freqüências esperadas sob o método da máxima verossimilhança, eleições 2002 . . . . . . . . . . . . . . . . 36

3.6 Evolução das freqüências esperadas sob o método bayesiano, eleições

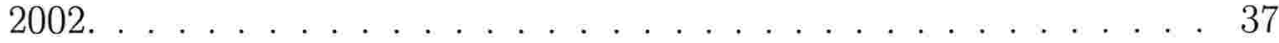




\section{Lista de Tabelas}

2.1 Número de alunos - consumo de drogas - USP . . . . . . . . . . . . . 5

2.2 Número de eleitores - eleições 2002 - segundo turno . . . . . . . . . . 6

3.1 Probabilidades - dados completos . . . . . . . . . . 16

3.2 Valores observados . . . . . . . . . . . . . . . . . 16

3.3 Probabilidades - dados incompletos. . . . . . . . . . . . . 17

3.4 Estimativas de $\theta$ sob o modelo ignorável (MAR ou MCAR) . . . . . 17

3.5 Estimativa de $\lambda$ sob o modelo MAR . . . . . . . . . . . . . 18

3.6 Valores observados dos testes em função de $\alpha$ para $S=10.000 \ldots 20$

3.7 Número de alunos com dados imputados - consumo de drogas - USP . 20

3.8 Freqüências observadas . . . . . . . . . . . . . . . . . . . . . . . . 24

3.9 Freqüências esperadas com a introdução da variável $R$. . . . . . . 28

3.10 Percentuais na intenção de voto - eleições 2002 - segundo turno . . . . 33

3.11 Intenções de voto com dados imputados - eleições 2002 - segundo turno 37

3.12 Percentuais na intenção de voto - eleições 2002 - segundo turno . . . . 38

C.1 Freqüências esperadas com a introdução da variável $R$. . . . . . . . . 54

D.1 Valores de $\alpha \ldots \ldots \ldots$. . . . . . . . . . . . . . . . . . . . 69 


\section{Capítulo 1}

\section{Introdução}

Independentemente da área de interesse, dados incompletos ou censurados são, com freqüência, um problema no momento de se efetuar uma análise de dados. A falta de dados eleva consideravelmente o nível de complexidade da análise e, como resultado, muitos analistas acabam dando pouca importância ao problema, e sub avaliando seu impacto. Além disso, falhas para identificar corretamente sua influência podem causar viés significativo, prejudicando, se não invalidando completamente, os resultados.

Dados censurados podem ocorrer por uma série de razões. Nas pesquisas, por exemplo, indivíduos podem achar certas perguntas irrelevantes, deixando de respondê-las. Além disso, alguns indivíduos podem simplesmente se recusar a responder certos tipos de perguntas. Existem ainda armadilhas estritamente técnicas, associadas a bancos de dados corrompidos, à fusão de dados de várias fontes e a erros de registro, que podem levar à eliminação de alguns dados.

Independentemente da razão, a ocorrência de dados censurados exige que o pesquisador leve em consideração algumas informações adicionais. Em muitas situações, a identificação das variáveis que explicam a causa dos dados censurados pode ajudar a reduzir o viés. Pode-se tomar como exemplo a situação dos indivíduos com muitos recursos econômicos, os quais podem não responder perguntas sobre renda. 
Tais variáveis, que podem explicar a causa dos dados perdidos, são conhecidas como "variáveis de mecanismo", e sua inclusão no modelo, pode reduzir (ou até mesmo eliminar) o viés causado por alguns tipos de dados perdidos. De fato, teoricamente, se todas as variáveis de mecanismo associadas a um conjunto particular de dados perdidos pudessem ser identificadas e incluídas como controles em um modelo, o impacto dos dados perdidos poderia ser, estatisticamente, ajustado até o ponto em que seja desprezível (Little e Rubin, 1987). Na prática, porém, é extremamente improvável que variáveis de mecanismo sejam identificadas para todos os casos de dados perdidos.

Uma situação de grande interesse está relacionada com dados categorizados. Muitas vezes, têm-se grupos de indivíduos que, por um motivo qualquer, não foram classificados por completo, sendo necessário alocá-los, de alguma forma, nas classes com maior probabilidade de pertinência. Esse procedimento, conhecido como imputação de dados categorizados, é o principal objetivo deste trabalho. Essa imputação terá como origem o modelo de censura que melhor se ajusta aos dados observados.

Uma outra abordagem considerada aqui diz respeito ao problema dos dados esparsos, que ocorrem com freqüência quando se está considerando dados incompletos.

Este trabalho é desenvolvido da seguinte forma: no Capítulo 2, são apresentados os problemas vinculados a dados incompletos, ilustrados por dois exemplos práticos. O Capítulo 3 aborda a imputação de dados categorizados, com base nos mecanismos de não-resposta, dentro do contexto do modelo probabilístico multinomial. No Capítulo 4 são apresentadas as principais conclusões obtidas. 


\section{Capítulo 2}

\section{Apresentação do Problema}

\subsection{Dados Observados}

Objetivando ilustrar este trabalho, foram selecionadas duas aplicações. A primeira está voltada para o mecanismo de censura ignorável vinculado ao problema dos dados esparsos. A segunda está direcionada para o mecanismo de censura nãoignorável.

Aplicação 1. Em uma pesquisa realizada entre alunos da USP foram obtidos dados relativos ao consumo das mais variadas drogas nos últimos 12 meses (Stempliuk, 2002). Nessa pesquisa, as drogas consumidas variavam desde drogas lícitas, como o tabaco e o álcool, até as ditas ilícitas, tais como a cocaína e o crack. Um caso de interesse corresponde ao consumo de álcool e cocaína categorizado por sexo. A Tabela 2.1, que contém dados da pesquisa, apresenta o inconveniente de que algumas celas possuem freqüências observadas muito baixas ( $\leq 5$ ou até zero), caracterizando a ocorrência de dados esparsos. Além disso, alguns alunos omitiram as respostas relativas ao consumo de álcool e/ou cocaína e assim a tabela também apresenta dados incompletos.

Nesta aplicação, foi adotado o mecanismo de censura ignorável, apesar de haver a possibilidade de se tentar ajustar o modelo não-ignorável. Na realidade, talvez 
este fosse o modelo mais adequado, pois é possível que a perda de informação sobre o uso de cocaína dependa de seu consumo. Entretanto, o objetivo principal nesta aplicação é mostrar como ajustar o modelo de censura ignorável quando, dentro de um grupo de variáveis categorizadas, apenas uma variável é sempre conhecida. Também são mostrados alguns métodos para o tratamento das celas com valores de freqüência muito baixos ou zero.

Tabela 2.1: Número de alunos - consumo de drogas - USP

\begin{tabular}{ccccc}
\hline & & \multicolumn{3}{c}{ Cocaína } \\
\cline { 3 - 5 } Sexo & Álcool & Sim & Não & S/R \\
\hline \multirow{2}{*}{ M } & Sim & 46 & 1265 & 21 \\
& Não & 0 & 276 & 3 \\
& S/R & 0 & 4 & 16 \\
\hline \multirow{2}{*}{ F } & Sim & 19 & 894 & 18 \\
& Não & 0 & 247 & 1 \\
& S/R & 0 & 2 & 11 \\
\hline
\end{tabular}

Fonte: Stempliuk (2002). S/R: sem resposta.

Aplicação 2. Pesquisa de intenção de voto espontânea para presidente no segundo turno das eleições 2002 realizada pelo Datafolha, em dois períodos no mês de outubro posteriores ao primeiro turno. Foram realizadas 10.397 entrevistas para o primeiro período e 10.377 para o segundo período. Segundo informações na página do Datafolha "a pesquisa Datafolha é um levantamento por amostragem estratificada por sexo e idade com sorteio aleatório dos entrevistados. O conjunto de eleitores acima de 16 anos do país é tomado como universo da pesquisa e dividido em quatro sub-universos que representam as regiões Sul, Sudeste, Nordeste e Norte/Centro-Oeste.

Em cada sub-universo, os municípios são agrupados de acordo com a localização e o nível sócio-econômico. Dentro de cada grupo são sorteados municípios estratificados pelo porte correspondente. Através de um processo de sorteios sucessivos, chega-se ao bairro, à rua e ao indivíduo." 
O primeiro período corresponde às pesquisas realizadas no dia 18 de outubro e o segundo no dia 26 de outubro. A Tabela 2.2 apresenta os resultados obtidos para dois níveis de idade: até 34 anos e acima de 34 anos.

As estimativas publicadas das proporções de votos válidos para os candidatos Lula e Serra foram respectivamente de $66 \%$ e $34 \%$ para o período 1 e de $64 \%$ e $36 \%$ para o período 2. O esquema amostral, embora importante, não foi considerado pois estamos interessados apenas em ilustrar como o modelo de censura pode ser ajustado.

Tabela 2.2: Número de eleitores - eleições 2002 - segundo turno

\begin{tabular}{ccccc}
\hline \multirow{2}{*}{ Período } & Idade & Lula & Serra & Sem Resposta \\
\cline { 3 - 5 } 1 & Até 34 anos & 2961 & 1436 & 236 \\
& Mais 34 anos & 3386 & 1890 & 488 \\
\hline \multirow{2}{*}{2} & Até 34 anos & 2815 & 1406 & 394 \\
& Mais 34 anos & 3198 & 1914 & 650 \\
\hline
\end{tabular}

Fonte: Datafolha.

\subsection{Mecanismos de Censura}

A nomenclatura utilizada para os mecanismos de censura varia entre os autores, de maneira que, neste trabalho, não nos deteremos numa delas, objetivando criar familiaridade com as de uso mais comum. Assim, mecanismos de censura podem também ser chamados de mecanismos de não-resposta e, dados perdidos (dependendo do contexto) podem ser chamados de dados censurados, omissos, incompletos, ausentes ou faltantes.

Dados incompletos são comuns na prática e pode ser necessário realizar estudos sobre o comportamento da perda desses dados ou informações. O uso de métodos que possibilitem identificar dados ou informações perdidas, viabilizando uma análise completa dos dados, é essencial para se alcançar conclusões válidas. A escolha do 
método apropriado depende das suposições feitas sobre o mecanismo de censura.

Little e Rubin (1987) dividem estes mecanismos em três categorias: perdas completamente ao acaso (missing completely at random-MCAR); perdas ao acaso (missing at random -MAR) e perdas não aleatórias (not missing at random -NMAR). Estes mecanismos podem ser "Ignoráveis", compreendidos pelos mecanismos MCAR e MAR e "Não-Ignoráveis", representados pelo mecanismo NMAR.

Os dados perdidos são considerados MCAR quando as "perdas" não estão relacionadas às variáveis especificadas. Esta é a mais restritiva das três condições no sentido que impõe que a probabilidade de não-resposta seja a mesma para diversas situações.

Os dados perdidos são considerados MAR, quando o padrão de perda é previsível a partir de outras variáveis no banco de dados em lugar de ser devido à variável específica na qual os dados são perdidos. Por exemplo, se os participantes de uma pesquisa que examina o comportamento temporal do nível de ansiedade como uma função da auto-estima, estiverem classificados na categoria baixa auto-estima, então têm menor probabilidade de devolverem os questionários para as sessões de seguimento do estudo. Se o investigador mediu auto-estima na sessão inicial, poderá então usá-la para predizer o padrão de perda dos dados.

Outro exemplo está relacionado com a compreensão de leitura. Os pesquisadores podem administrar um teste de compreensão de leitura no início da pesquisa. Os participantes com escores de compreensão de leitura mais baixos podem ser menos propícios a completar a pesquisa inteira. Nestes exemplos, as variáveis para as quais os dados são perdidos não são a causa dos dados omitidos, a qual é devida a alguma outra influência externa (baixa compreensão de leitura). Por exemplo, na Aplicação 2 (Tabela 2.2), existem perdas de informação sobre a variável Intenção de Voto; se o mecanismo é MAR, o padrão de perda poderia ser explicado pelas variáveis Idade e Período.

Finalmente, os dados perdidos são considerados NMAR quando as perdas não ocorrem de forma aleatória. Em contraste com o mecanismo MAR, situação es- 
boçada anteriormente, onde a perda de dados é explicável através de outras variáveis medidas em um estudo, os dados perdidos NMAR surgem devido ao padrão de perda que é explicável pela(s) mesma(s) variável(is) para a(s) qual(is) os dados são perdidos. Pode-se tomar como exemplo um estudo sobre a renda de indivíduos de diversos níveis sociais. Se interpretarmos a informação sobre a renda, reduzida ao fato de essa informação existir ou não, como uma variável resposta dicotômica, podemos sugerir que, caso haja interação entre a variável resposta dicotômica apresentada e a variável renda, teremos indícios de um mecanismo de censura NMAR. Em contrapartida, se essa interação não for detectada, há indícios de que o mecanismo de censura seja ignorável. De fato, essa é a condição de dados perdidos mais difícil de se lidar e, infelizmente, é a mais provável em situações reais. 


\section{Capítulo 3}

\section{Imputação de Dados}

\section{Categorizados sob o Modelo}

\section{Probabilístico Multinomial}

\subsection{Mecanismo de Não-Resposta Ignorável}

Vamos assumir, por simplicidade, que em uma determinada pesquisa existam três variáveis e que as variáveis 1,2 e 3 tenham I, J e K níveis, indexados por $i, j$ e $k$, respectivamente. Os resultados aqui apresentados podem ser generalizados para problemas com maior número de variáveis.

Consideremos uma amostra com dados censurados. O Modelo Multinomial para a situação hipotética na qual todos os elementos estariam completamente classificados é dado por:

$$
p(\mathbf{n} \mid N, \boldsymbol{\theta})=N ! \prod_{i} \prod_{j} \prod_{k} \frac{\theta_{i j k}^{n_{i j k}}}{n_{i j k} !}
$$

em que $\sum_{i j k} \theta_{i j k}=1 \mathrm{e}$

$\theta_{i j k}=$ probabilidade de que uma unidade amostral escolhida ao acaso tenha sido 
classificada na cela $(i j k)$;

$N=$ número total de unidades amostrais;

$n_{i j k}=$ freqüência hipoteticamente observada na cela $i j k$;

$\mathrm{n}=$ vetor de freqüências hipoteticamente observadas $n_{i j k}$;

$\theta=$ vetor de probabilidades $\theta_{i j k}$.

Para dados incompletos, usando a notação de Willamson e Haber (1994), tomemos $\lambda_{i j}^{12}$ como a probabilidade de ausência do valor da variável 3. De forma análoga, tomemos $\lambda_{j k}^{23}$ e $\lambda_{i k}^{13}$ como as probabilidades de omissão dos valores das variáveis $1 \mathrm{e}$ 2 , respectivamente e, $\lambda_{i}^{1}, \lambda_{j}^{2}$ e $\lambda_{k}^{3}$ como as probabilidades de omissão das variáveis 2 e 3,1 e 3 e 1 e 2 , respectivamente. A probabilidade de omissão de todas as variáveis é zero, restrição necessária para que o modelo seja identificável. Finalmente, a probabilidade de não haver omissão de qualquer das variáveis (caso da classificação completa) é:

$$
\mu_{i j k}=1-\left(\lambda_{i j}^{12}+\lambda_{j k}^{23}+\lambda_{i k}^{13}+\lambda_{i}^{1}+\lambda_{j}^{2}+\lambda_{k}^{3}\right)
$$

e assim temos que

$$
0 \leq\left(\lambda_{i j}^{12}+\lambda_{j k}^{23}+\lambda_{i k}^{13}+\lambda_{i}^{1}+\lambda_{j}^{2}+\lambda_{k}^{3}\right) \leq 1
$$

para todo $i, j, k$.

Admitindo-se que pelo menos uma determinada variável seja sempre observada (a variável 1), tem-se que

$$
\lambda_{j k}^{23}=\lambda_{j}^{2}=\lambda_{k}^{3}=0
$$

e assim,

$$
\mu_{i j k}=1-\left(\lambda_{i j}^{12}+\lambda_{i k}^{13}+\lambda_{i}^{1}\right)
$$

$\mathrm{e}$

$$
0 \leq\left(\lambda_{i j}^{12}+\lambda_{i k}^{13}+\lambda_{i}^{1}\right) \leq 1 .
$$

Para os dados verdadeiramente observados consideremos o modelo multinomial: 
$p(\mathbf{z}, \mathbf{u} \mid N, \boldsymbol{\theta}, \lambda)=N ! \prod_{i j k} \frac{\left(\mu_{i j k} \theta_{i j k}\right)^{z_{i j k}}}{z_{i j k} !} \prod_{i j} \frac{\left(\lambda_{i j}^{12} \theta_{i j+}\right)^{u_{i j}^{12}}}{u_{i j}^{12} !} \prod_{i k} \frac{\left(\lambda_{i k}^{13} \theta_{i+k}\right)^{u_{i k}^{13}}}{u_{i k}^{13} !} \prod_{i} \frac{\left(\lambda_{i}^{1} \theta_{i++}\right)^{u_{i}^{1}}}{u_{i}^{1} !}$

em que

$z_{i j k}=$ freqüência observada na cela $i j k$ correspondente às unidades amostrais completamente classificadas;

$u_{i j}^{12}=$ freqüência observada correspondente às unidades amostrais com ausência de informação sobre a variável 3 ;

$u_{i k}^{13}=$ freqüência observada correspondente às unidades amostrais com ausência de informação sobre a variável 2 ;

$u_{i}^{1}=$ freqüência observada correspondente às unidades amostrais com ausência de informação sobre as variáveis 2 e 3 ;

$\mathbf{z}=$ vetor de freqüências completamente observadas $z_{i j k}$;

$\mathbf{u}=$ vetor de freqüências com classificação incompleta;

$Z=$ número de unidades amostrais completamente observadas;

$U=$ número de unidades amostrais com classificação incompleta (a serem imputadas), tais que $Z+U=N$.

O índice + indica o somatório das probabilidades nas categorias da.(s) variável(is) correspondente(s).

Para exemplificar, consideremos a Aplicação 1. A notação, para os dados das Tabelas 2.1 e 3.3, é:

$z_{112}$ : número observado de alunos do sexo masculino, consumidores de álcool e não-consumidores de cocaína (1265);

$z_{221}$ : número observado de alunos do sexo feminino, não-consumidores de álcool e consumidores de cocaína (0);

$u_{12}^{13}$ : número observado de alunos do sexo masculino, não-consumidores de cocaína, 
com a informação sobre o consumo de álcool ausente (4);

$u_{2}^{1}$ : número observado de alunos do sexo feminino, com informações sobre o consumo de álcool e de cocaína ausentes (11).

A notação para $\lambda$ é similar àquela para $\mathbf{u}$, mas diz respeito a probabilidades em vez de freqiiências observadas. Por exemplo,

$\lambda_{11}^{12}$ : probabilidade de que um aluno do sexo masculino, consumidor de álcool, tenha omitida a informação sobre o consumo de cocaína.

Na Aplicação $1, Z=2747, U=76, N=2823$.

\subsubsection{Estimação dos Parâmetros}

Levando-se em conta que a distribuição multinomial pertence à família exponencial e tomando-se o modelo saturado, pode-se usar o algoritmo EM como segue.

Com base na expressão (3.2) vislumbra-se a função de verossimilhança, a qual pode ser fatorada e escrita como o produto de dois termos, um que só depende de $\theta$ e outro que só depende de $\boldsymbol{\lambda}$. As estimativas de $\boldsymbol{\theta}$ e $\boldsymbol{\lambda}$ são obtidas a partir do termo correspondente. Os detalhes dessa fatoração podem ser encontrados no Apêndice A.

\section{Estimativa de $\theta$}

As estimativas de $\theta$ serão as mesmas para os modelos MAR e MCAR, pois a diferença entre eles está nas restrições impostas sobre $\lambda$, as quais não afetam $\theta$.

Os passos do algoritmo EM são obtidos da seguinte forma:

Passo E:

$$
Q\left(\boldsymbol{\theta}, \boldsymbol{\theta}^{(g)}\right)=E\left\{\ln [p(\boldsymbol{\theta} \mid \mathbf{n})] \mid \mathbf{z}, \mathbf{u}, \boldsymbol{\theta}^{(g)}\right\}
$$

em que

$$
\ln [p(\theta \mid \mathrm{n})]=\ln N !+\sum_{i} \sum_{j} \sum_{k} n_{i j k} \ln \theta_{i j k}-\sum_{i} \sum_{j} \sum_{k} \ln n_{i j k}
$$


ou seja,

$$
\ln [p(\theta \mid \mathbf{n})] \propto \sum_{i} \sum_{j} \sum_{k} n_{i j k} \ln \theta_{i j k}
$$

Então,

$$
\begin{aligned}
& Q\left(\boldsymbol{\theta}, \theta^{(g)}\right)=E\left\{\sum_{i} \sum_{j} \sum_{k} n_{i j k} \ln \theta_{i j k} \mid \mathbf{z}, \mathbf{u}, \theta^{(g)}\right\} \\
& Q\left(\theta, \theta^{(g)}\right)=\sum_{i} \sum_{j} \sum_{k} E\left\{n_{i j k} \mid \mathbf{z}, \mathbf{u}, \theta^{(g)}\right\} \ln \theta_{i j k},
\end{aligned}
$$

sendo $g$ o índice da iteração.

A partir dessa expressão e levando-se em conta que $\hat{n}_{i j k}^{(g)}=E\left\{n_{i j k} \mid \mathrm{z}, \mathbf{u}, \boldsymbol{\theta}^{(g)}\right\}$ obtemos

$$
\hat{n}_{i j k}^{(g)}=z_{i j k}+\frac{u_{i j}^{12}}{\hat{\theta}_{i j+}^{\hat{\hat{n}}}} \hat{\theta}_{i j k}^{(g)}+\frac{u_{i k}^{13}}{\hat{\theta}_{i+k}^{(g)}} \hat{\theta}_{i j k}^{(g)}+\frac{u_{i}^{1}}{\hat{\theta}_{i++}^{(g)}} \hat{\theta}_{i j k}^{(g)} .
$$

\section{Passo M:}

Deve-se encontrar o valor de $\boldsymbol{\theta}$ que maximize $Q\left(\boldsymbol{\theta}, \boldsymbol{\theta}^{(g)}\right)$. De acordo com os detalhes apresentados no Apêndice A, esse valor pode ser escrito como:

$$
\hat{\theta}_{i j k}^{(g+1)}=\frac{\hat{n}_{i j k}^{(g)}}{N} .
$$

\section{Estimativa de $\lambda$}

Para a determinação das estimativas de máxima verossimilhança das probabilidades de omissão de alguma(s) variável(is), $\boldsymbol{\lambda}$, deve-se encontrar o valor representado por $\hat{\lambda}$, que maximiza a função $\ln L(\lambda)=\ell(\lambda)$.

As expressões envolvendo os elementos de $\lambda$ são:

$$
\begin{aligned}
\frac{u_{i j}^{12}}{\lambda_{i j}^{12}} & =\sum_{k}\left\{\frac{z_{i j k}}{1-\lambda_{i j}^{12}-\lambda_{i k}^{13}-\lambda_{i}^{1}}\right\}, \\
\frac{u_{i k}^{13}}{\lambda_{i k}^{13}} & =\sum_{j}\left\{\frac{z_{i j k}}{1-\lambda_{i j}^{12}-\lambda_{i k}^{13}-\lambda_{i}^{1}}\right\} \mathrm{e} \\
\frac{u_{i}^{1}}{\lambda_{i}^{1}} & =\sum_{j} \sum_{k}\left\{\frac{z_{i j k}}{1-\lambda_{i j}^{12}-\lambda_{i k}^{13}-\lambda_{i}^{1}}\right\} .
\end{aligned}
$$


Essas expressões compõem um sistema de equações não-lineares cujas soluções correspondem às estimativas de máxima verossimilhança de $\lambda$, aqui obtidas pelo método de Newton-Raphson.

Maiores detalhes sobre a estimação dos parâmetros $\theta$ e $\lambda$ são apresentados no Apêndice A.

\section{Variâncias de $\hat{\theta}$ e $\hat{\lambda}$}

As matrizes de covariância assintótica podem ser estimadas a partir da matriz de informação de Fisher, substituindo-se $\boldsymbol{\theta}$ e $\boldsymbol{\lambda}$ por $\hat{\boldsymbol{\theta}}$ e $\hat{\boldsymbol{\lambda}}$, respectivamente, ou seja,

$$
\begin{aligned}
& \widehat{\operatorname{Var}}(\hat{\boldsymbol{\theta}})=\left[-\mathbf{E}\left(\frac{\partial^{2} \ell(\boldsymbol{\theta})}{\partial \boldsymbol{\theta} \partial \boldsymbol{\theta}^{\top}}\right)\right]_{\hat{\theta}}^{-1} \mathrm{e} \\
& \widehat{\operatorname{Var}}(\hat{\boldsymbol{\lambda}})=\left[-\mathbf{E}\left(\frac{\partial^{2} \ell(\boldsymbol{\lambda})}{\partial \boldsymbol{\lambda} \partial \boldsymbol{\lambda}^{\top}}\right)\right]_{\hat{\boldsymbol{\lambda}}}^{-\mathbf{1}}
\end{aligned}
$$

Maiores detalhes sobre a obtenção dessas matrizes de covariância podem ser vistos no Apêndice B.

\subsubsection{Ajuste de Modelos Estruturais}

O objetivo da análise está diretamente relacionado com a simplificação da estrutura dos parâmetros do modelo adotado. Tal simplificação vai ao encontro de determinados anseios por parte do pesquisador quanto à verificação do comportamento de determinado fenômeno. Esse comportamento pode ser ajustado ao modelo através dessa simplificação estrutural, que corresponde a determinadas restrições impostas aos parâmetros do modelo saturado.

Dentre os modelos estruturais, pode-se citar, como exemplo, os modelos de simetria, de homogeneidade marginal e de independência, entre outros (Agresti, 1990).

Os estimadores obtidos através do que foi proposto na Seção 3.1.1 correspondem aos de um determinado modelo MAR saturado, ou seja, àquele modelo MAR que se 
ajusta perfeitamente aos dados. Temos interesse em ajustar o modelo MCAR, ou seja, a situação em que a perda de dados é totalmente aleatória.

\section{Ajuste do Modelo MCAR}

Como foi dito anteriormente, o modelo saturado é o modelo com mecanismo de censura MAR que se ajusta perfeitamente aos dados. Para o ajuste do modelo sob censura MCAR será necessário impor restrições ao parâmetro $\lambda$. Esse parâmetro representa a probabilidade da ausência de informação de determinada(s) variável(is) do estudo e, conseqüentemente, a imposição de restrições sobre ele viabiliza a verificação de determinadas condições comuns entre seus elementos. A situação MCAR é representada pela restrição de igualdade de parâmetros correspondente à idêntica probabilidade de ausência de variável(is) do estudo, ou seja, o modelo MCAR deve respeitar a restrição sob a qual todos os parâmetros $\lambda_{i j}^{12}$ são iguais para todo $i, j$. O mesmo deve ocorrer para os parâmetros $\lambda_{i k}^{13}$ para todo $i, k$ e para os parâmetros $\lambda_{i}^{1}$ para todo $i$.

O ajuste do modelo pode ser testado por intermédio dos já bem conhecidos testes de Pearson ou da razão de verossimilhanças, descritos no Apêndice D, Seção D.2.

\subsubsection{Aplicação}

Retomando o problema do consumo de drogas (Tabela 2.1) podemos estar interessados em verificar o ajuste do modelo MCAR.

A situação hipotética na qual todas as observações da amostra estariam com as informações completas sobre cada variável está representada na Tabela 3.1, que contém as probabilidades das observações serem classificadas nas celas.

A situação real, quanto às freqüências observadas, pode ser representada pela Tabela 3.2 .

De forma equivalente, pode-se representar a Tabela 3.2 em função dos parâmetros 
Tabela 3.1: Probabilidades - dados completos

\begin{tabular}{cccc}
\hline & & \multicolumn{2}{c}{ Cocaína } \\
\cline { 3 - 4 } Sexo & Álcool & $\operatorname{Sim}$ & Não \\
\hline & Sim & $\theta_{111}$ & $\theta_{112}$ \\
M & Não & $\theta_{121}$ & $\theta_{122}$ \\
\hline & Sim & $\theta_{211}$ & $\theta_{212}$ \\
F & Não & $\theta_{221}$ & $\theta_{222}$ \\
\hline
\end{tabular}

Tabela 3.2: Valores observados

\begin{tabular}{ccccc}
\hline & & \multicolumn{3}{c}{ Cocaína } \\
\cline { 3 - 5 } Sexo & Álcool & Sim & Não & S/R \\
\hline \multirow{2}{*}{ M } & Sim & $z_{111}$ & $z_{112}$ & $u_{11}^{12}$ \\
& Não & $z_{121}$ & $z_{122}$ & $u_{12}^{12}$ \\
& S/R & $u_{11}^{13}$ & $u_{12}^{13}$ & $u_{1}^{1}$ \\
\hline \multirow{2}{*}{ F } & Sim & $z_{211}$ & $z_{212}$ & $u_{21}^{12}$ \\
& Não & $z_{221}$ & $z_{222}$ & $u_{22}^{12}$ \\
& S/R & $u_{21}^{13}$ & $u_{22}^{13}$ & $u_{2}^{1}$ \\
\hline
\end{tabular}

S/R: Sem resposta.

$\boldsymbol{\theta}$ e $\boldsymbol{\lambda}$ (Tabela 3.3).

\section{Estimativas de $\theta$ e $\lambda$}

As estimativas de $\theta$ sob o modelo MAR saturado, obtidas por intermédio do algoritmo EM e o aplicativo S-Plus, cujo programa correspondente encontra-se no Apêndice A, estão na Tabela 3.4. Como as freqüências observadas $z_{121}$ e $z_{221}$ são zero, as estimativas de $\theta_{121}$ e $\theta_{221}$ também o são, o que impossibilita obter a inversa da matriz de informação de Fisher. Para viabilizar a obtenção da estimativa do erro padrão (quinta coluna da Tabela 3.4), foi adotado o valor 0,0001 como estimativa para $\theta_{121}$ e $\theta_{221}$. Para mero efeito comparativo, foram obtidas as proporções $p_{i j k}$ com base na subamostra composta pelas freqüências observadas $z_{i j k}$ de tal forma que $p_{i j k}=\frac{z_{i j k}}{Z}$ (Tabela 3.4). No caso do modelo MCAR ser adequado, deve-se 
Tabela 3.3: Probabilidades - dados incompletos.

\begin{tabular}{ccccc}
\hline & & \multicolumn{3}{c}{ Cocaína } \\
\cline { 3 - 5 } Sx & Ĺle & $\operatorname{Sim}$ & Não & Sem Resposta \\
\hline & Sim & $\left(1-\lambda_{11}^{12}-\lambda_{11}^{13}-\lambda_{1}^{1}\right) \theta_{111}$ & $\left(1-\lambda_{11}^{12}-\lambda_{12}^{13}-\lambda_{1}^{1}\right) \theta_{112}$ & $\lambda_{11}^{12}\left(\theta_{111}+\theta_{112}\right)$ \\
M & Não & $\left(1-\lambda_{12}^{12}-\lambda_{11}^{13}-\lambda_{1}^{1}\right) \theta_{121}$ & $\left(1-\lambda_{12}^{12}-\lambda_{12}^{13}-\lambda_{1}^{1}\right) \theta_{122}$ & $\lambda_{12}^{12}\left(\theta_{121}+\theta_{122}\right)$ \\
& S/R & $\lambda_{11}^{13}\left(\theta_{111}+\theta_{121}\right)$ & $\lambda_{12}^{13}\left(\theta_{112}+\theta_{122}\right)$ & $\lambda_{1}^{1}\left(\theta_{111}+\theta_{112}+\theta_{121}+\theta_{122}\right)$ \\
\hline & Sim & $\left(1-\lambda_{21}^{12}-\lambda_{21}^{13}-\lambda_{2}^{1}\right) \theta_{211}$ & $\left(1-\lambda_{21}^{12}-\lambda_{22}^{13}-\lambda_{2}^{1}\right) \theta_{212}$ & $\lambda_{21}^{12}\left(\theta_{211}+\theta_{212}\right)$ \\
F & Não & $\left(1-\lambda_{22}^{12}-\lambda_{21}^{13}-\lambda_{2}^{1}\right) \theta_{221}$ & $\left(1-\lambda_{22}^{12}-\lambda_{22}^{13}-\lambda_{2}^{1}\right) \theta_{222}$ & $\lambda_{22}^{12}\left(\theta_{221}+\theta_{222}\right)$ \\
& S/R & $\lambda_{21}^{13}\left(\theta_{211}+\theta_{221}\right)$ & $\lambda_{22}^{13}\left(\theta_{212}+\theta_{222}\right)$ & $\lambda_{2}^{1}\left(\theta_{211}+\theta_{212}+\theta_{221}+\theta_{222}\right)$ \\
\hline
\end{tabular}

S/R: Sem resposta.

esperar que $\hat{\theta}_{i j k}=p_{i j k}$.

Tabela 3.4: Estimativas de $\theta$ sob o modelo ignorável (MAR ou MCAR)

\begin{tabular}{c|cc|cc|cc}
\hline \multirow{2}{*}{ Celas ijk } & \multicolumn{2}{|c|}{ Dados Completos } & \multicolumn{2}{c|}{ EM } & \multicolumn{2}{c}{ EM $(\alpha=0,32)$} \\
\cline { 2 - 7 } & $p_{i j k}$ & e.p. $\left(\times 10^{-3}\right)$ & $\hat{\theta}_{i j k}$ & e.p. $\left(\times 10^{-3}\right)$ & $\tilde{\theta}_{i j k}$ & e.p. $\left(\times 10^{-3}\right)$ \\
\hline 111 & 0,01675 & 2,45 & 0,01670 & 2,46 & 0,01693 & 2,48 \\
112 & 0,46050 & 9,51 & 0,46097 & 12,80 & 0,46041 & 12,78 \\
121 & 0,00000 & 0,12 & 0,00010 & 54,00 & 0,00012 & 0,21 \\
122 & 0,10047 & 5,74 & 0,10007 & 6,02 & 0,10013 & 5,98 \\
211 & 0,00692 & 1,58 & 0,00693 & 1,59 & 0,00715 & 1,61 \\
212 & 0,32545 & 8,94 & 0,32650 & 10,78 & 0,32622 & 10,76 \\
221 & 0,00000 & 0,12 & 0,00010 & 66,34 & 0,00012 & 0,21 \\
222 & 0,08992 & 4,72 & 0,08882 & 5,65 & 0,08892 & 5,63 \\
\hline
\end{tabular}

Observa-se na Tabela 3.4 que as estimativas dos erros-padrão de $\hat{\theta}_{121}$ e $\hat{\theta}_{221}$ são muito maiores do que as de $p_{121}$ e $p_{221}$, respectivamente. Essa situação é criada por causa das celas com freqüência zero ou muito baixas. Uma solução para isso, conforme descrição na Seção D.3 do Apêndice D, é adicionar uma constante $\alpha$ a cada cela da tabela. Usando-se $\alpha=0,32$ (cuja escolha está justificada adiante), nota-se que esse problema é reduzido significativamente, como pode ser observado nas colunas seis e sete da Tabela 3.4, justificando a importância do procedimento 
de adição de constantes.

Maiores detalhes a respeito da obtenção das estimativas dos erros-padrão podem ser vistos no Apêndice $B$, bem como o programa correspondente, desenvolvido para o aplicativo MatLab.

A Tabela 3.5 mostra os valores obtidos para as estimativas dos parâmetros $\lambda$ 's e respectivos erros-padrão sob o modelo MAR. Para se evitar falha na convergência pelo método de Newton-Raphson e também para viabilizar a obtenção das estimativas dos erros-padrão, foi adicionada a constante $\alpha=0,32$ em todas as celas. Os programas para obtenção dessas estimativas foram desenvolvidos para os aplicativos Mathematica e MatLab e encontram-se nos Apêndices A e B, respectivamente.

Tabela 3.5: Estimativa de $\boldsymbol{\lambda}$ sob o modelo MAR

\begin{tabular}{ccc}
\hline Estimador & Estimativa $\left(\times 10^{-3}\right)$ & e.p. $\left(\times 10^{-3}\right)$ \\
\hline$\hat{\lambda}_{1}^{1}$ & 9,99 & 2,48 \\
$\hat{\lambda}_{11}^{12}$ & 15,56 & 3,35 \\
$\hat{\lambda}_{12}^{12}$ & 11,71 & 6,39 \\
$\hat{\lambda}_{11}^{13}$ & 6,64 & 11,70 \\
$\hat{\lambda}_{12}^{13}$ & 2,72 & 1,31 \\
$\hat{\lambda}_{21}^{12}$ & 19,09 & 4,42 \\
$\hat{\lambda}_{22}^{12}$ & 5,24 & 5,22 \\
$\hat{\lambda}_{21}^{13}$ & 15,58 & 27,32 \\
$\hat{\lambda}_{22}^{13}$ & 1,98 & 1,30 \\
$\hat{\lambda}_{2}^{1}$ & 9,48 & 2,80 \\
\hline
\end{tabular}

\section{Ajuste do Modelo MCAR}

O modelo saturado com relação a $\boldsymbol{\lambda}$ é o modelo com mecanismo de censura MAR. Dessa maneira, a imposição de restrições ao modelo de não-resposta ignorável leva ao mecanismo de censura MCAR. Com esse objetivo, será necessário impor restrições do tipo $\lambda_{11}^{12}=\lambda_{12}^{12}=\lambda_{21}^{12}=\lambda_{22}^{12}, \lambda_{11}^{13}=\lambda_{12}^{13}=\lambda_{21}^{13}=\lambda_{22}^{13}$ e $\lambda_{1}^{1}=\lambda_{2}^{1}$ ao parâmetro

$$
\boldsymbol{\lambda}=\left(\begin{array}{llllllllll}
\lambda_{1}^{1} & \lambda_{11}^{12} & \lambda_{12}^{12} & \lambda_{11}^{13} & \lambda_{12}^{13} & \lambda_{21}^{12} & \lambda_{22}^{12} & \lambda_{21}^{13} & \lambda_{22}^{13} & \lambda_{2}^{1}
\end{array}\right)^{\top} .
$$


A aplicação do teste do modelo MCAR versus o modelo MAR leva ao valor da estatística da razão de verossimilhanças de $Q_{V}=5,034$ e da estatística de Pearson de $Q_{P}=3,982$, correspondentes a p-valores de 0,656 e 0,782 , respectivamente.

Dessa forma, os testes fornecem fortes evidências de que o modelo MCAR pode ser bem ajustado aos dados quando comparado ao modelo MAR . Entretanto, ocorre uma diferença nos valores obtidos para os p-valores dos testes, em torno de $20 \%$ maior no teste de Pearson. Isso pode estar relacionado ao fato de algumas celas apresentarem freqüências zero ou baixas sendo necessário algum método que amenize esses efeitos tais como os apresentados no Apêndice D, Seção D.3.

Para essa aplicação foi utilizado o procedimento da adição de uma constante às observações das celas, tanto para o teste de Pearson como para o teste da razão de verossimilhanças. A Tabela 3.6 apresenta os p-valores obtidos diretamente e por intermédio da simulação da distribuição empírica do valor das estatísticas dos testes. A Figura 3.1 mostra a curva formada pelos p-valores (razão de verossimilhanças e Pearson) em função de $\alpha$.

As Figuras 3.2 e 3.3 mostram os histogramas construídos a partir dos valores dos testes de Pearson e de Wilks das amostras geradas para diversos valores de $\alpha$. Para um valor de $\alpha$ em torno de 0,32 o histograma apresenta um perfil muito próximo da curva gerada pela distribuição $\chi^{2}$ com 7 graus de liberdade. Na Figura 3.1, quando o valor de $\alpha$ está em torno de 0,32 ocorre uma situação na qual o p-valor obtido pela simulação é praticamente igual a 0,67 tanto para o teste de Pearson como para o teste da razão de verossimilhanças. Seria razoável adotar esse valor para $\alpha$ tendo em vista que, em torno dele, o p-valor permanece praticamente constante para o teste de Pearson e é decrescente para o teste da razão de verossimilhanças (uma vez que tende, nessa situação, a ser conservador). De qualquer forma, um p-valor dessa ordem mantém a decisão de não rejeitar o modelo MCAR.

Aplicando as restrições nas expressões (3.5), obtém-se a estimativa do vetor $\lambda$ sob o modelo MCAR:

$$
\hat{\lambda}_{11}^{12}=\hat{\lambda}_{12}^{12}=\hat{\lambda}_{21}^{12}=\hat{\lambda}_{22}^{12}=0,0152,
$$




$$
\begin{aligned}
& \hat{\lambda}_{11}^{13}=\hat{\lambda}_{12}^{13}=\hat{\lambda}_{21}^{13}=\hat{\lambda}_{22}^{13}=0,0021 \mathrm{e} \\
& \hat{\lambda}_{1}^{1}=\hat{\lambda}_{2}^{1}=0,0096 .
\end{aligned}
$$

Tabela 3.6: Valores observados dos testes em função de $\alpha$ para $S=10.000$

\begin{tabular}{|c|c|c|c|c|c|c|}
\hline \multirow{2}{*}{$\alpha$} & \multicolumn{2}{|c|}{ Valor do Teste } & \multicolumn{2}{c|}{ p-valor } & \multicolumn{2}{c|}{ p-valor (simulação) } \\
\cline { 2 - 7 } & Pearson & RV & Pearson & RV & Pearson & RV \\
\hline 0,01 & 3,80 & 4,71 & 0,80 & 0,70 & 0,48 & 0,61 \\
\hline 0,10 & 3,58 & 4,32 & 0,83 & 0,74 & 0,63 & 0,72 \\
\hline 0,20 & 3,86 & 4,32 & 0,80 & 0,74 & 0,67 & 0,73 \\
\hline 0,32 & 4,79 & 4,56 & 0,69 & 0,71 & 0,67 & 0,67 \\
\hline 0,35 & 5,11 & 4,64 & 0,65 & 0,70 & 0,66 & 0,64 \\
\hline 0,50 & 7,10 & 5,18 & 0,42 & 0,64 & 0,64 & 0,52 \\
\hline 1,00 & 16,90 & 7,78 & 0,02 & 0,35 & 0,48 & 0,18 \\
\hline 2,00 & 41,70 & 14,50 & 0,00 & 0,04 & 0,25 & 0,03 \\
\hline$\left(^{*}\right)$ & 3,98 & 5,03 & 0,78 & 0,66 & - & - \\
\hline
\end{tabular}

$(*)$ Valor do teste aplicado diretamente, sem adição de $\alpha$.

A Tabela 3.7 apresenta as freqüências obtidas para o modelo ignorável (MAR ou MCAR) após a imputação. Esses resultados são obtidos mantendo-se as freqüências marginais correspondentes aos dados incompletos e por isso são iguais para os dois modelos.

Tabela 3.7: Número de alunos com dados imputados - consumo de drogas - USP

\begin{tabular}{cccc}
\hline & & \multicolumn{2}{c}{ Cocaína } \\
\cline { 3 - 4 } Sexo & Álcool & Sim & Não \\
\hline & Sim & 48 & 1300 \\
M & Não & 0 & 283 \\
\hline & Sim & 20 & 921 \\
F & Não & 0 & 251 \\
\hline
\end{tabular}




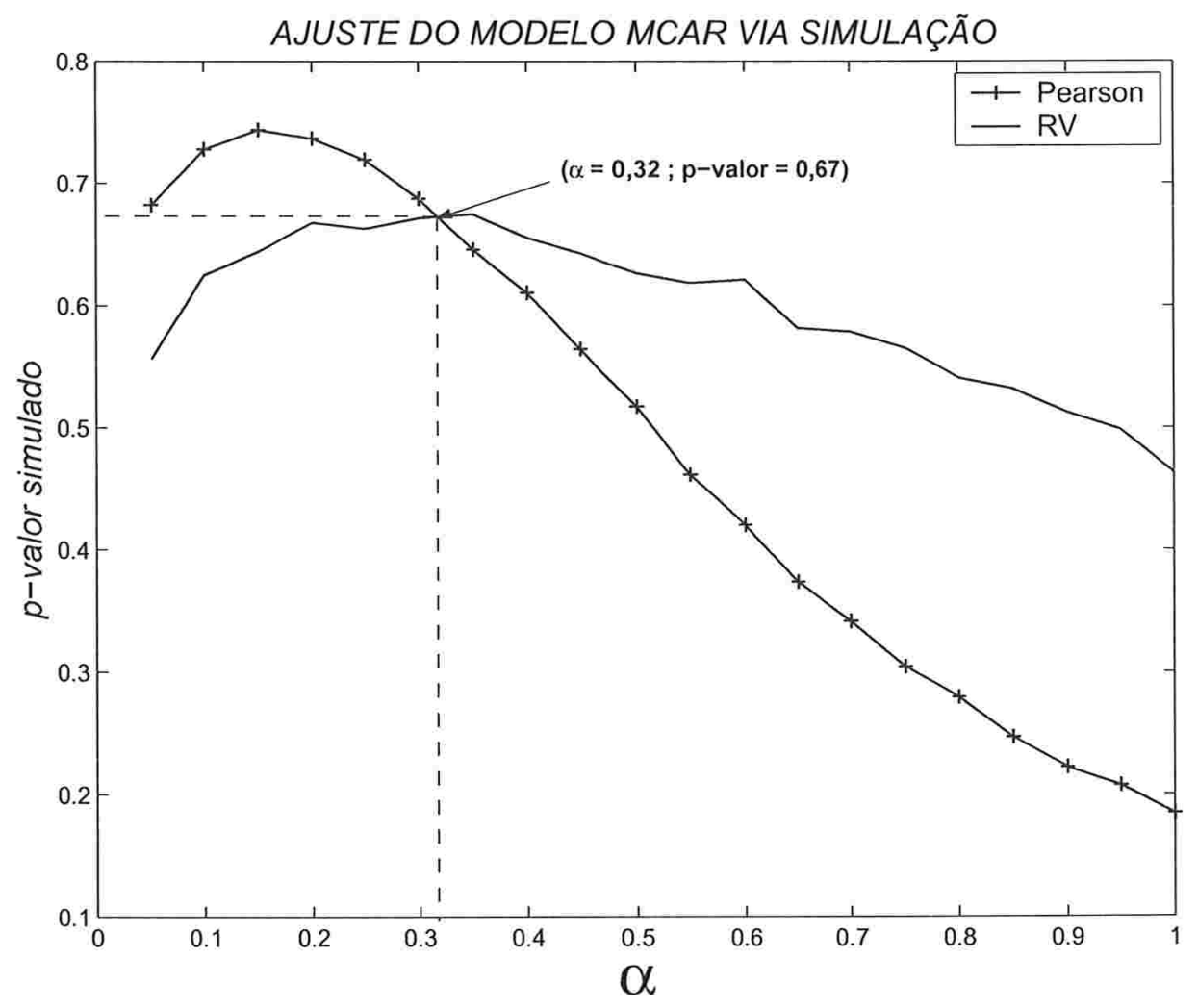

Figura 3.1: Gráfico indicando a variação do p-valor simulado em função da variação de $\alpha$. 

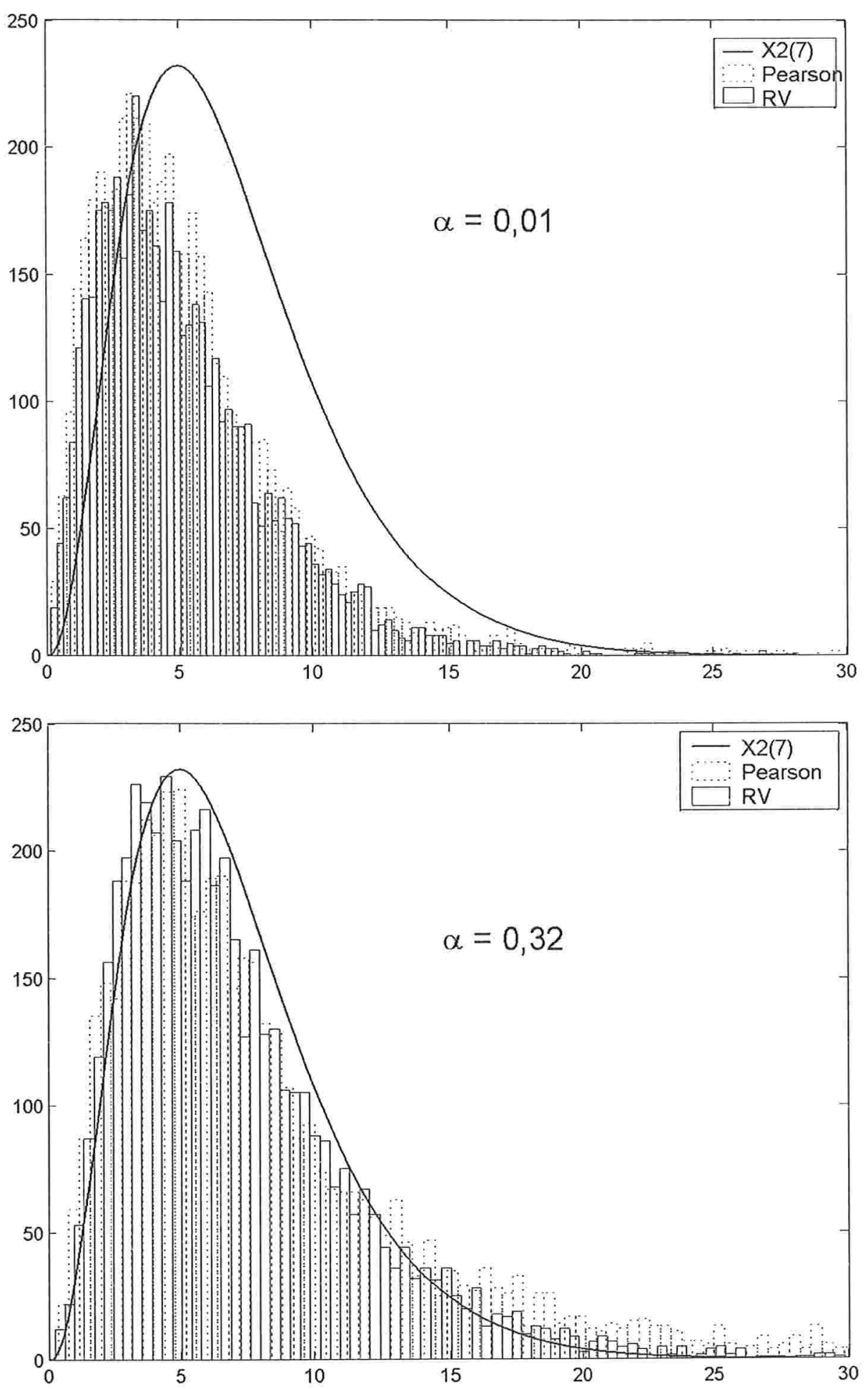

Figura 3.2: Gráficos representando as distribuições dos valores dos testes simulados de Pearson e RV para $\alpha$ igual a 0,01 e 0,32 juntamente com a distribuição $\chi^{2}$ com 7 graus de liberdade. 

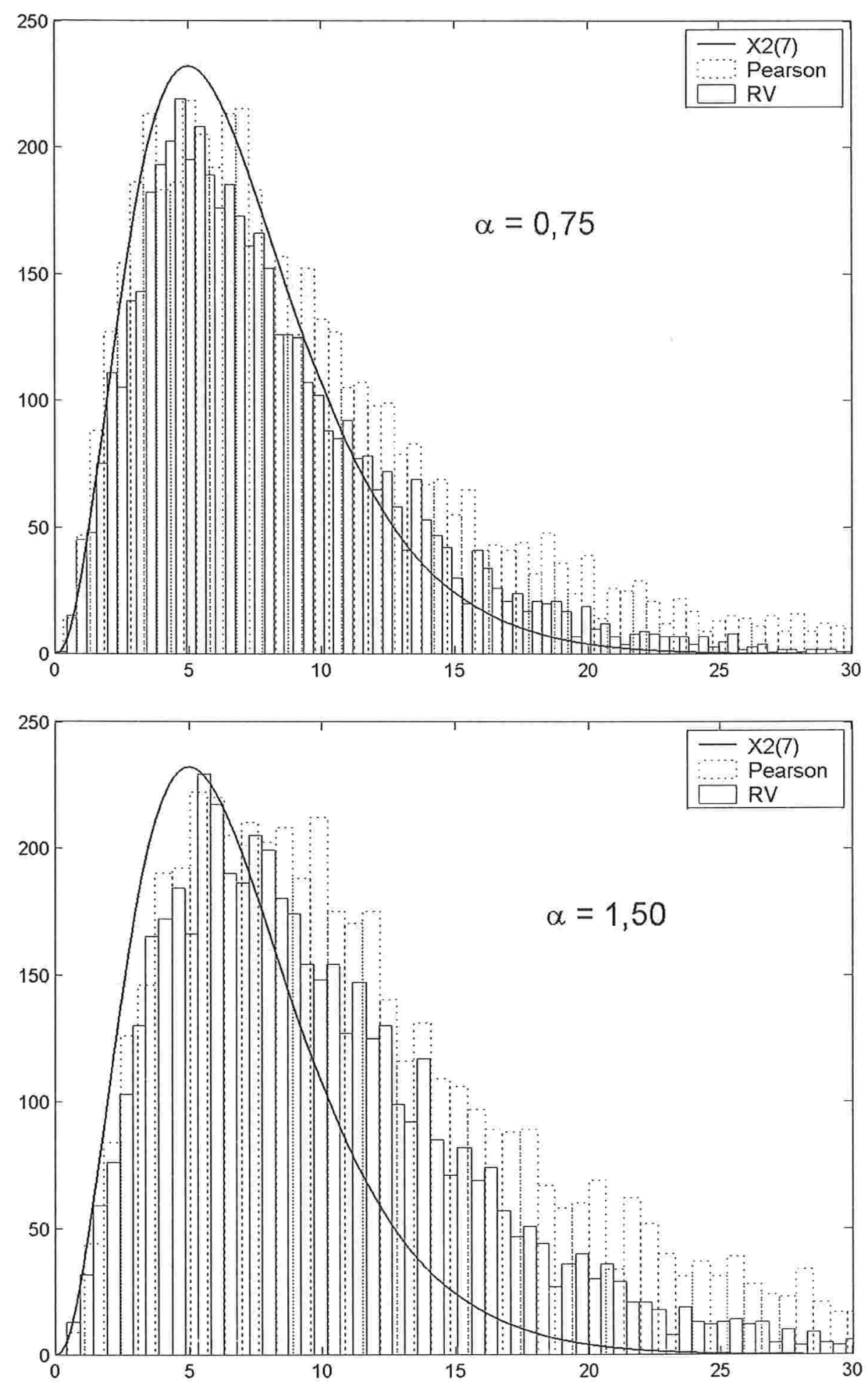

Figura 3.3: Gráficos representando as distribuições dos valores dos testes simulados de Pearson e RV para $\alpha$ igual a 0,75 e 1,50 juntamente com a distribuição $\chi^{2}$ com 7 graus de liberdade. 


\subsection{Mecanismo de Não-Resposta Não-Ignorável}

Uma classe de modelos log-lineares, proposta por Fay (1986) e Baker e Laird (1988), pode assumir o mecanismo de censura não-ignorável para dados categorizados. Nesta seção usaremos este tipo de modelo, que é ajustado a tabelas de dados aumentados nas quais uma variável dicotômica é incluída, indicando se a observação de determinada variável ocorreu ou não.

Devido à complexidade na aplicação do modelo log-linear para uma tabela de contingência de três entradas, quando mais do que uma variável apresenta dados ausentes, optou-se, neste trabalho, pela ilustração baseada em uma situação específica. Vamos considerar aqui uma tabela tridimensional (Tabela 3.8) composta por uma variável resposta Y indexada por $j$, que poderá conter informações omissas e um vetor bidimensional, $\mathbf{X}$, composto pelas variáveis explicativas $X_{1}$ e $X_{2}$, sempre observadas, indexado por $\mathbf{i}=\left(i_{1}, i_{2}\right)$. Seja $J$ o número de categorias de $\mathrm{Y}$ e $I_{1}$ e $I_{2}$ o número de categorias de $X_{1}$ e $X_{2}$, respectivamente. Uma variável indicadora dicotômica $\mathrm{R}$, indexada por $k$, será introduzida objetivando apontar se a variável $\mathrm{Y}$ está sendo informada ou não. Quando $R=1$, $Y$ foi observado e quando $R=2$, não há informação sobre Y.

Tabela 3.8: Freqüências observadas

\begin{tabular}{ccccc}
\hline & & \multicolumn{3}{c}{$Y$} \\
\cline { 3 - 5 }$X_{1}$ & $X_{2}$ & 1 & 2 & Sem Resp. \\
\hline 1 & 1 & $n_{(11) 11}$ & $n_{(11) 21}$ & $n_{(11)+2}$ \\
& 2 & $n_{(12) 11}$ & $n_{(12) 21}$ & $n_{(12)+2}$ \\
\hline 2 & 1 & $n_{(21) 11}$ & $n_{(21) 21}$ & $n_{(21)+2}$ \\
& 2 & $n_{(22) 11}$ & $n_{(22) 21}$ & $n_{(22)+2}$ \\
\hline
\end{tabular}

Os índices na notação utilizada na Tabela 3.8 indicam o seguinte: os dois números entre parêntesis indicam os níveis de $X_{1}$ e $X_{2}$; os demais indicam os níveis de $Y$ e $R$, respectivamente. Por exemplo, $n_{(12) 21}$ é o número de observações no nível 1 de $X_{1}$, nível 2 de $X_{2}$ e nível 2 de $Y$. Quando a informação sobre $Y$ é desconhecida, o 
índice correspondente a essa variável é substituído por "+".

Montando-se a tabela de contingência de maneira que todas as celas apresentem informações de $\mathrm{X}, Y$ e $R$ então, um modelo de não-resposta será não-ignorável quando possuir um termo de interação entre as variáveis $Y$ e $R$.

\subsubsection{O Modelo Log-linear}

Um modelo $\log$-linear pode assumir a forma $\ln \mathrm{m}=\mathrm{Z} \beta$ em que $\mathrm{m}$ é o vetor de freqüências esperadas $m_{\mathrm{i} j k}$ sob o modelo, Z é uma matriz de especificação de dimensão $c \times p$ onde $c$ é o número de celas e $p$ o número de parâmetros do modelo de tal forma que $\boldsymbol{\beta}=\left(\beta_{1}, \beta_{2}, \ldots, \beta_{p}\right)$ é o vetor de parâmetros com $p \leq c$. A notação ln $\mathrm{m}$ indica o vetor de dimensão $c \times 1$ em que cada componente é o logaritmo natural do correspondente componente em $\mathrm{m}$.

Com a introdução da variável indicadora $R$, o modelo log-linear apresentando todos os parâmetros possíveis pode ser escrito como

$$
\begin{aligned}
\ln m_{\left(i_{1} i_{2}\right) j k}= & \beta_{0}+\beta_{i_{1}}^{X_{1}}+\beta_{i_{2}}^{X_{2}}+\beta_{j}^{Y}+\beta_{k}^{R}+\beta_{i_{1} i_{2}}^{X_{1} X_{2}}+\beta_{i_{1} j}^{X_{1} Y}+\beta_{i_{1} k}^{X_{1} R}+\beta_{i_{2} j}^{X_{2} Y}+\beta_{i_{2} k}^{X_{2} R} \\
& +\beta_{j k}^{Y R}+\beta_{i_{1} i_{2} j}^{X_{1} X_{2} Y}+\beta_{i_{1} i_{2} k}^{X_{1} X_{2} R}+\beta_{i_{1} j k}^{X_{1} Y R}+\beta_{i_{2} j k}^{X_{2} Y R}+\beta_{i_{1} i_{2} j k}^{X_{1} X_{2} Y R} .
\end{aligned}
$$

com as restrições usuais:

$$
\begin{aligned}
\sum_{i_{1}} \beta_{i_{1}}^{X_{1}} & =\sum_{i_{2}} \beta_{i_{2}}^{X_{2}}=\sum_{j} \beta_{j}^{Y}=\sum_{k} \beta_{k}^{R}=0 \\
\sum_{i_{1}} \beta_{i_{1} i_{2}}^{X_{1} X_{2}} & =\sum_{i_{2}} \beta_{i_{1} i_{2}}^{X_{1} X_{2}}=\sum_{i_{1}} \beta_{i_{1} j}^{X_{1} Y}=\sum_{j} \beta_{i_{1} j}^{X_{1} Y}=0 \\
\sum_{i_{1}} \beta_{i_{1} k}^{X_{1} R} & =\sum_{k} \beta_{i_{1} k}^{X_{1} R}=\sum_{i_{2}} \beta_{i_{2} j}^{X_{2} Y}=\sum_{j} \beta_{i_{2} j}^{X_{2} Y}=0 \\
\sum_{i_{2}} \beta_{i_{2} k}^{X_{2} R} & =\sum_{k} \beta_{i_{2} k}^{X_{2} R}=\sum_{j} \beta_{j k}^{Y R}=\sum_{k} \beta_{j k}^{Y R}=0 \\
\sum_{i_{1}} \beta_{i_{1} i_{2} j}^{X_{1} X_{2} Y} & =\sum_{i_{2}} \beta_{i_{1} i_{2} j}^{X_{1} X_{2} Y}=\sum_{j} \beta_{i_{1} i_{2} j}^{X_{1} X_{2} Y}=\sum_{i_{1}} \beta_{i_{1} i_{2} k}^{X_{1} X_{2} R}=0 \\
\sum_{i_{2}} \beta_{i_{1} i_{2} k}^{X_{1} X_{2} R} & =\sum_{k} \beta_{i_{1} i_{2} k}^{X_{1} X_{2} R}=\sum_{i_{1}} \beta_{i_{1} j k}^{X_{1} Y R}=\sum_{j} \beta_{i_{1} j k}^{X_{1} Y R}=0
\end{aligned}
$$




$$
\begin{aligned}
\sum_{k} \beta_{i_{1} j k}^{X_{1} Y R} & =\sum_{i_{2}} \beta_{i_{2} j k}^{X_{2} Y R}=\sum_{j} \beta_{i_{2} j k}^{X_{2} Y R}=\sum_{k} \beta_{i_{2} j k}^{X_{2} Y R}=0 \\
\sum_{i_{1}} \beta_{i_{1} i_{2} j k}^{X_{1} X_{2} Y R} & =\sum_{i_{2}} \beta_{i_{1} i_{2} j k}^{X_{1} X_{2} Y R}=\sum_{j} \beta_{i_{1} i_{2} j k}^{X_{1} X_{2} Y R}=\sum_{k} \beta_{i_{1} i_{2} j k}^{X_{1} X_{2} Y R}=0
\end{aligned}
$$

para todo $i_{1}, i_{2}, j$ e $k$.

Esse modelo servirá como base para a imposição dos dois mecanismos de censura aqui tratados.

\section{Ignorável}

Dentro do contexto de modelos hierárquicos e sob o mecanismo de censura ignorável (representado pelos modelos MAR), o modelo log-linear apropriado pode ser expresso como

$$
\begin{aligned}
\ln m_{\left(i_{1} i_{2}\right) j k}= & \beta_{0}+\beta_{i_{1}}^{X_{1}}+\beta_{i_{2}}^{X_{2}}+\beta_{j}^{Y}+\beta_{k}^{R}+\beta_{i_{1} i_{2}}^{X_{1} X_{2}}+\beta_{i_{1} j}^{X_{1} Y}+\beta_{i_{1} k}^{X_{1} R}+\beta_{i_{2} j}^{X_{2} Y}+\beta_{i_{2} k}^{X_{2} R} \\
& +\beta_{i_{1} i_{2} j}^{X_{1} X_{2} Y}+\beta_{i_{1} i_{2} k}^{X_{1} X_{2} R} .
\end{aligned}
$$

Esse modelo pode ser escrito na notação log-linear como $\left(X_{1} X_{2} Y, X_{1} X_{2} R\right)$ com as restrições usuais de identificabilidade. Nessa situação tem-se $Y$ e $R$ condicionalmente independentes, dados $X_{1}$ e $X_{2}$. Há ausência da interação de primeira ordem $Y R$, das interações de segunda ordem $X_{1} Y R$ e $X_{2} Y R$ e da interação de terceira ordem $X_{1} X_{2} Y R$. Assim, o modelo não permite a interação entre as variáveis $Y$ e $R$, caracterizando a situação em que a probabilidade de censura depende dos dados observados e não depende dos dados que foram perdidos.

\section{Não-Ignorável}

Um mecanismo de censura não-ignorável é representado por:

$$
\begin{aligned}
\ln m_{\left(i_{1} i_{2}\right) j k}= & \beta_{0}+\beta_{i_{1}}^{X_{1}}+\beta_{i_{2}}^{X_{2}}+\beta_{j}^{Y}+\beta_{k}^{R}+\beta_{i_{1} i_{2}}^{X_{1} X_{2}}+\beta_{i_{1} j}^{X_{1} Y}+\beta_{i_{1} k}^{X_{1} R}+\beta_{i_{2} j}^{X_{2} Y} \\
& +\beta_{j k}^{Y R}+\beta_{i_{1} i_{2} j}^{X_{1} X_{2} Y}+\beta_{i_{1} j k}^{X_{1} Y R}
\end{aligned}
$$

escrito como $\left(X_{1} X_{2} Y, X_{1} Y R\right)$, também com as restrições usuais. Aqui, $X_{2}$ e $R$ são condicionalmente independentes dado $X_{1}$ e $Y$. Nessa situação, ocorreria a ausência da interação de primeira ordem $X_{2} R$, das interações de segunda ordem $X_{1} X_{2} R$ e 
$X_{2} Y R$ e da interação de terceira ordem $X_{1} X_{2} Y R$. A proposta desse modelo está voltada para a interação entre as variáveis resposta $Y$ e $R$, significando agora que a probabilidade de censura depende dos dados ausentes.

\subsubsection{Estimação dos Parâmetros}

Os estimadores de máxima verossimilhança para as freqüências nas celas podem ser obtidos pela maximização da função de verossimilhança correspondente ao modelo log-linear restrito, usando-se o algoritmo EM.

\section{Máxima Verossimilhança}

O núcleo da função de log-verossimilhança de uma densidade conjunta de $\mathrm{X}$ e $Y$ condicionada em $R$ pode ser escrito como

$$
\ell=\sum_{\mathbf{i}} \sum_{j} n_{\mathbf{i} j 1} \ln \left(\theta_{\mathbf{i} j \mid 1}\right)+\sum_{\mathbf{i}} n_{\mathbf{i}+2} \ln \left(\theta_{\mathbf{i}+\mid 2}\right),
$$

em que $\theta_{\mathbf{i} j \mid 1}=p[\mathbf{X}=\mathbf{i}, Y=j \mid R=1], \theta_{\mathbf{i}+\mid 2}=\sum_{j} p[\mathbf{X}=\mathbf{i}, Y=j \mid R=2], n_{\mathbf{i j} 1}$ são as freqüências observadas para os dados completamente informados e $n_{\mathbf{i}+2}$ são as freqüências para os dados com ausência de informação sobre $Y$.

A Tabela 3.9 apresenta as freqüências esperadas com a introdução da variável $R$.

Os elementos do vetor de probabilidades $\theta$ são parametrizados como

$$
\theta_{\mathbf{i} j \mid k}=\frac{m_{\mathbf{i} j k}}{m_{++k}} .
$$

A função de verossimilhança é maximizada sob freqüências pseudo-observadas nas celas, representadas por $n_{\mathrm{i} j 2}^{*}$, fazendo-se uso do algoritmo $E M$. O passo $E$ do algoritmo determina as freqüências pseudo-observadas nas celas e o passo $M$ obtém os estimadores de máxima verossimilhança dessas freqüências. Dentro do enfoque do modelo de censura não-ignorável, a estimação de máxima verossimilhança pode levar a soluções que se encontram nas bordas do espaço paramétrico, quando algumas estimativas de $m_{\mathbf{i} j 2}$ são zeros. Quando essas soluções limites ocorrem, as estimativas 
Tabela 3.9: Freqüências esperadas com a introdução da variável $R$

\begin{tabular}{ccccc}
\hline & & & \multicolumn{2}{c}{$R$} \\
\cline { 3 - 5 }$X_{1}$ & $X_{2}$ & $\mathrm{Y}$ & $\operatorname{Sim}$ & Não \\
\hline 1 & 1 & 1 & $m_{(11) 11}$ & $m_{(11) 12}$ \\
& & 2 & $m_{(11) 21}$ & $m_{(11) 22}$ \\
\cline { 3 - 5 } & 2 & 1 & $m_{(12) 11}$ & $m_{(12) 12}$ \\
& & 2 & $m_{(12) 21}$ & $m_{(12) 22}$ \\
\hline 2 & 1 & 1 & $m_{(21) 11}$ & $m_{(21) 12}$ \\
& & 2 & $m_{(21) 21}$ & $m_{(21) 22}$ \\
\cline { 3 - 5 } & 2 & 1 & $m_{(22) 11}$ & $m_{(22) 12}$ \\
& & 2 & $m_{(22) 21}$ & $m_{(22) 22}$ \\
\hline
\end{tabular}

dos parâmetros não podem ser determinadas de forma única. Esta instabilidade é causada pelo ajuste do modelo incluindo termos de interação YR. Com o objetivo de contornar esses problemas, foi proposta uma abordagem bayesiana por Park e Brown (1994).

\section{Método Bayesiano}

Em uma abordagem bayesiana, simplificada para estimar os parâmetros em um modelo de regressão logística, Clogg et al. (1991) propuseram usar uma constante de ajuste, de maneira que o valor de maximização seja único. A partir dessa abordagem, Park e Brown (1994) usaram uma priori com uma distribuição de Dirichlet para $\theta_{\mathrm{i} j \mid 2}$ na forma conjugada,

$$
\prod_{i} \prod_{j} e^{a j p}
$$

em que $\alpha_{\mathrm{i} j}$ são constantes de ajuste positivas a serem especificadas (análogas às constantes $\alpha$ da Seção 3.1.3). O resultado posterior a uma transformação logarítmica é

$$
p(\theta)=\sum_{\mathrm{i}} \sum_{j} n_{\mathrm{i} j 1} \ln \left(\theta_{\mathrm{i} j \mid 1}\right)+\sum_{\mathrm{i}}\left[n_{\mathrm{i}+2} \ln \left(\theta_{\mathrm{i}+\mid 2}\right)+\sum_{j} \alpha_{\mathrm{i} j} \ln \left(\theta_{\mathbf{i} j \mid 2}\right)\right]
$$




$$
=\sum_{\mathbf{i}} \sum_{j} n_{\mathbf{i} j 1} \ln \frac{m_{\mathbf{i} j 1}}{m_{++1}}+\sum_{\mathbf{i}}\left[n_{\mathbf{i}+2} \ln \frac{m_{\mathbf{i}+2}}{m_{++2}}+\sum_{j} \alpha_{\mathbf{i} j} \ln \frac{m_{\mathbf{i} j 2}}{m_{++2}}\right]
$$

que também pode ser escrita como:

$$
\begin{aligned}
p(\theta)= & \sum_{\mathbf{i}} \sum_{j} n_{\mathrm{i} j 1}\left[\ln \left(m_{\mathrm{i} j 1}\right)-\ln \left(m_{++1}\right)\right]+\sum_{\mathbf{i}}\left[n_{i+2}\left[\ln \left(m_{\mathrm{i}+2}\right)-\ln \left(m_{++2}\right)\right]+\right. \\
& \left.\sum_{j} \alpha_{\mathbf{i} j}\left[\ln \left(m_{\mathrm{i} j 2}\right)-\ln \left(m_{++2}\right)\right]\right] .
\end{aligned}
$$

Seja $\mathbf{z}_{\mathbf{i} j k}$ a linha de $\mathbf{Z}$ correspondente a $m_{\mathbf{i} j k}$ e $z_{(\mathbf{i} j k) v}$ o v-ésimo elemento de $\mathbf{z}_{\mathbf{i} j k}$. Dessa maneira, $p(\boldsymbol{\theta})$ pode ser expresso em função de $\boldsymbol{\beta}$ como:

$$
\begin{aligned}
p(\boldsymbol{\theta})= & \sum_{\mathbf{i}} \sum_{j} n_{\mathbf{i} j 1}\left(\mathbf{z}_{\mathbf{i} j 1} \boldsymbol{\beta}\right)-n_{++1} \ln \sum_{\mathbf{i}} \sum_{j} \exp \left(\mathbf{z}_{\mathbf{i} j 1} \boldsymbol{\beta}\right)+ \\
& \sum_{\mathbf{i}}\left\{n_{\mathbf{i}+2} \ln \left[\sum_{j} \exp \left(\mathbf{z}_{\mathbf{i} j 2} \boldsymbol{\beta}\right)\right]+\sum_{j} \alpha_{\mathrm{i} j} \ln \left(\mathbf{z}_{\mathbf{i} j 2} \boldsymbol{\beta}\right)\right\}- \\
& \left(n_{++2}+\Delta\right) \ln \sum_{\mathbf{i}} \sum_{j} \exp \left(\mathbf{z}_{\mathbf{i} j 2} \boldsymbol{\beta}\right),
\end{aligned}
$$

em que

$$
\Delta=\sum_{\mathbf{i}} \sum_{j} \alpha_{\mathbf{i} j}
$$

Derivando-se $p(\boldsymbol{\theta})$ com relação a cada um dos elementos de $\boldsymbol{\beta}$, representados por $\beta_{v}, v=1, \ldots, p$, e expressando o resultado em termos de $m_{\mathbf{i} j k}$, tem-se

$$
\sum_{\mathbf{i}} \sum_{j} z_{(\mathbf{i} j 1) v}\left(n_{\mathbf{i} j 1}-m_{\mathbf{i} j 1}\right)+\sum_{\mathbf{i}} \sum_{j} z_{(\mathbf{i} j 2) v}\left\{n_{\mathbf{i}+2} \frac{m_{\mathbf{i} j 2}}{m_{\mathbf{i}+2}}+\alpha_{\mathbf{i} j}-m_{\mathbf{i} j 2}\right\}=0 .
$$

Definindo

$$
\begin{aligned}
& n_{\mathrm{i} j 1}^{*}=E\left(n_{\mathrm{i} j 1}\right)=n_{\mathrm{i} j 1} \\
& n_{\mathrm{i} j 2}^{*}=E\left(n_{\mathrm{i} j 2}\right)=n_{\mathrm{i}+2} \frac{m_{\mathrm{i} j 2}}{m_{\mathbf{i}+2}}+\alpha_{\mathrm{i} j}
\end{aligned}
$$

temos

$$
\sum_{\mathbf{i}} \sum_{j} z_{(\mathrm{i} j 1) v}\left(n_{\mathrm{i} j 1}-m_{\mathrm{i} j 1}\right)+\sum_{\mathbf{i}} \sum_{j} z_{(\mathrm{i} j 2) v}\left\{n_{\mathrm{i} j 2}^{*}\left[1+p \frac{n_{\mathrm{i}+1}}{n_{\mathrm{i}+2} n_{++1}}\right]-m_{\mathrm{i} j 2}\right\}=0 .
$$

Sob o enfoque bayesiano, as estimativas de máxima posteriori (EMP) das freqüências esperadas nas celas são obtidas a partir das freqüências pseudo-observadas $n_{\mathrm{i} j 2}^{*}$ que 
maximizam o logaritmo da posteriori sob as condições $m_{++1}=n_{++1}$ e $m_{++2}=$ $n_{++2}+\Delta$.

Essas estimativas podem ser obtidas pelo uso das equações (3.15) e (3.16) no passo $\mathrm{E}$ e a equação (3.17) no passo $\mathrm{M}$ até que um critério de convergência seja satisfeito.

Como o EMV não tem forma fechada, para obtê-lo no passo M é necessário o uso de algum algoritmo de maximização como por exemplo o IPF.

As Expressões

$$
\begin{array}{lll}
m_{\mathbf{i} j k}^{(3 g+1)} & =m_{\mathbf{i} j k}^{(3 g)} \frac{n_{\mathbf{i} j+}^{*}}{m_{\mathbf{i} j+}^{(3 g)}} & (X Y) \\
m_{\mathbf{i} j k}^{(3 g+2)} & =m_{\mathbf{i} j k}^{(3 g+1)} \frac{n_{\mathbf{i}+k}^{*}}{m_{\mathbf{i}+k}^{(3 g+1)}} & (X R) \\
m_{\mathbf{i} j k}^{(3 g+3)} & =m_{\mathbf{i} j k}^{(3 g+2)} \frac{n_{+j k}^{*}}{m_{+j k}^{(3 g+2)}} & (Y R)
\end{array}
$$

definem o algoritmo IPF para o passo M (Apêndice D, Seção D.1.4), que maximiza o modelo posterior de não-resposta impondo as freqüências pseudo-observadas, $n_{\mathrm{i} j 2}^{*}=$ $E\left(n_{\mathrm{i} j 2}\right)$, como sendo as freqüências observadas nas celas. Dependendo do modelo a ser ajustado, o passo M do algoritmo EM difere, ou seja, por exemplo, se o modelo a ser ajustado é $(X Y, X R)$, são usadas as Expressões (3.18) e (3.19). O algoritmo EM, usando-se o algoritmo IPF no passo $M$, sempre converge para uma solução, mesmo quando a solução é próxima de um limite do espaço paramétrico, quando toma-se o valor 0 para $\alpha_{\mathbf{i} j}$ na expressão (3.17) (Dempster, Laird e Rubin, 1977).

Embora a condição $\Delta=\sum_{\mathbf{i}} \sum_{j} \alpha_{\mathbf{i} j}$ seja usada para obter a equação (3.11), seria conveniente aplicar um fator de correção $(\varphi)$ em $n_{\mathbf{i} j 2}^{*}$ de maneira a satisfazer $m_{++2}=n_{++2}$. Esse procedimento leva em conta que $n_{\mathrm{i} j 2}^{*}$ é um valor imputado para a pseudo-freqüência observada na cela $n_{\mathbf{i} j 2}$ cujo total, $n_{\mathbf{i}+2}$, para um dado $\mathbf{i}$, é uma freqüência verdadeiramente observada. Tomando-se $\varphi=\frac{n_{i+2}}{n_{i+2}+\alpha_{i+}}$, pode-se garantir que $n_{\mathbf{i}+2}^{*}=n_{\mathbf{i}+2}$, o que implica que, para cada $\mathbf{i}$, a soma das freqüências pseudo-observadas é igual àquela das freqüências observadas nas celas, e a condição $m_{++2}=n_{++2}$ está satisfeita. Para atender a essas imposições, as freqüências pseudo- 
observadas nas celas $n_{\mathbf{i} j 2}^{*}$ são redefinidas como:

$$
n_{\mathbf{i} j 2}^{*}=\frac{n_{\mathbf{i}+2}}{n_{\mathbf{i}+2}+\alpha_{\mathbf{i}+}}\left[n_{\mathbf{i}+2} \frac{m_{\mathbf{i j} 2}}{m_{\mathbf{i}+2}}+\alpha_{\mathbf{i} j}\right] .
$$

Quando $\alpha_{\mathbf{i} j}=0$, a abordagem se reduz àquela proposta por Fay (1986) e Baker e Laird (1988), ou seja, corresponde ao método de máxima verossimilhança. Nessa abordagem, as freqüências pseudo-observadas nas celas são exclusivamente determinadas por $m_{\mathrm{i} j k}$. Uma vez que $m_{\mathrm{i} j k}$ seja estimada como zero, continuará como zero ao longo das iterações, o que poderá acarretar estimativas instáveis para os parâmetros $\beta$. A introdução de uma constante de ajuste, $\alpha_{\mathbf{i} j}$, pode evitar esse resultado indesejado; $n_{\mathrm{i} j k}^{*}$ sempre permanecerá positivo, desde que $n_{\mathrm{i} j k}$ seja positivo. Ao contrário da abordagem de máxima verossimilhança, essa abordagem pelo método bayesiano acarretará estimativas estáveis e únicas dos parâmetros do modelo.

Para especificar a distribuição a priori, é necessário determinar $\Delta$ e repartí-lo dentro dos valores de $\alpha_{\mathrm{i} j}$, ou seja, distribuir a quantidade $\Delta$ de forma a gerar as quantidades $\alpha_{\mathrm{i} j}$. Seguindo a abordagem de Clogg et al. (1991), $\Delta$ foi escolhido como sendo $p$, o número de parâmetros do modelo a serem estimados, pois este valor já corresponderia a uma situação de estabilidade nas estimativas das freqüências esperadas.

Sob o mecanismo de censura ignorável, o EMV de $m_{\mathrm{i} j 2}$ pode ser obtido como

$$
\hat{m}_{\mathbf{i} j 2}=n_{\mathbf{i}+2}\left(\frac{n_{\mathbf{i} j 1}}{n_{\mathbf{i}+1}}\right)
$$

e neste contexto, escolhe-se o $\alpha_{\mathbf{i} j}$ que melhor aproxima o EMP de $m_{\mathbf{i} j 2}$ dessas estimativas, ou seja, $\alpha_{\mathbf{i} j}$ é escolhido de forma a ser proporcional aos $n_{\mathbf{i} j 1}$. Tomando$\Delta=p$, temos

$$
\alpha_{\mathbf{i} j}=p \frac{n_{\mathbf{i} j 1}}{n_{++1}} .
$$

Dessa maneira, $n_{\mathbf{i} j 2}^{*}$ deve ser expresso como

$$
n_{\mathbf{i} j 2}^{*}=\frac{n_{\mathbf{i}+2} n_{++1}}{n_{\mathbf{i}+2} n_{++1}+p n_{\mathbf{i}+1}}\left[n_{\mathbf{i}+2} \frac{m_{\mathbf{i} j 2}}{m_{\mathbf{i}+2}}+p \frac{n_{\mathbf{i} j 1}}{n_{++1}}\right] .
$$


Assim a distribuição a priori (3.10) é dependente dos dados, uma vez que $\alpha_{\mathrm{i} j}$ é estimado a partir das freqüências observadas $n_{\mathrm{i} j 1}$.

Tomando-se $\alpha_{\mathrm{i} j}=0$ nas equações (3.12) e (3.16), obtêm-se as equações de verossimilhança:

$$
\begin{aligned}
n_{i j 1}+n_{i j 2}^{*} & =m_{i j+} \quad \forall i, j=1,2, \\
n_{+j 1}=m_{+j 1} \text { e } n_{+j 2}^{*} & =m_{+j 2} \quad, \quad j=1,2 .
\end{aligned}
$$

Geralmente, as soluções das equações (3.23) e (3.24) não possuem uma forma fechada e somente podem ser obtidas por um processo iterativo como, por exemplo, o algoritmo EM. Essas soluções dependem das variáveis

$$
v_{1}=\frac{n_{111}}{n_{+11}}, \quad v_{2}=\frac{n_{121}}{n_{+21}} \quad \text { e } \quad v_{3}=\frac{n_{1+2}}{n_{++2}} .
$$

Quando $v_{3}$ está entre $v_{1}$ e $v_{2}$, a estimação de máxima verossimilhanças decompõe os totais marginais dos dados censurados nas duas categorias de $Y$. No caso contrário, a tabela é completada pela alocação de todos os dados ausentes em somente uma das categorias. Nessa situação, as freqüências esperadas para as celas de uma das categorias de $Y$ são estimadas como zero, e os estimadores dos parâmetros $\boldsymbol{\beta}$ não podem ser unicamente determinados. Quando o procedimento bayesiano é aplicado e uma constante de ajuste $\alpha_{i j}$ é introduzida, evita-se esse problema, ou seja, uma solução de borda só ocorre quando $v_{3}$ está próximo de 0 ou 1.

\subsubsection{Aplicação}

Dentro do contexto proposto por Baker (1996), tomemos a Aplicação 2 das eleições (Tabela 2.2) e montemos a Tabela 3.10 em termos de valores percentuais fixada a faixa etária e o período.

Nota-se que, percentualmente, existe uma maior aproximação dos indivíduos com intenção de voto desconhecida com aqueles que possuem intenção de voto no candidato Serra para os dois períodos. Tal comportamento sugere que existe uma maior perda de informação de intenção de voto no grupo de indivíduos que votariam no 
Tabela 3.10: Percentuais na intenção de voto - eleições 2002 - segundo turno

\begin{tabular}{|c|c|c|c|c|}
\hline \multirow[b]{2}{*}{ Período } & \multirow[b]{2}{*}{ Idade } & \multicolumn{3}{|c|}{ Intenção de Voto } \\
\hline & & Lula & Serra & Sem resposta \\
\hline \multirow[t]{2}{*}{1} & Até 34 anos & $46,6 \%$ & $43,2 \%$ & $32,6 \%$ \\
\hline & Mais 34 anos & $53,4 \%$ & $56,8 \%$ & $67,4 \%$ \\
\hline \multirow[t]{2}{*}{2} & Até 34 anos & $46,8 \%$ & $42,4 \%$ & $37,7 \%$ \\
\hline & Mais 34 anos & $53,2 \%$ & $57,6 \%$ & $62,3 \%$ \\
\hline
\end{tabular}

candidato Serra, aumentando o potencial desse candidato. Uma conseqüência imediata desse comportamento é que as perdas de informação não ocorrem de forma aleatória, ou seja, o mecanismo de censura é não-ignorável. Assim, haveria, na verdade, um maior número de indivíduos, entre aqueles com voto desconhecido, que potencialmente votariam no candidato Serra do que haveria se o mecanismo fosse ignorável.

A aplicação aos dados das pesquisas eleitorais visa somente o aspecto ilustrativo e por isso a amostragem complexa realizada foi ignorada. No entanto, destacamos que esses dados merecem uma futura análise mais elaborada.

Nesta aplicação, o modelo adequado é o produto de multinomiais. Entretanto, se uma reparametrização adequada for feita, o modelo log-linear do produto de multinomiais pode ser formulado de maneira idêntica ao modelo log-linear correspondente ao modelo multinomial. Os novos parâmetros expressos com base nas probabilidades condicionais no contexto do produto de multinomiais têm forma análoga aos parâmetros expressos com base nas probabilidades conjuntas do modelo multinomial (Agresti, 1990).

Para exemplificar, consideremos o modelo antes da introdução da variável $R$. O modelo log-linear saturado no contexto multinomial, expresso com base no parâmetro $\theta$, pode ser escrito como:

$$
\ln \theta_{i_{1} i_{2} j}=\beta_{0}+\beta_{i_{1}}^{X_{1}}+\beta_{i_{2}}^{X_{2}}+\beta_{j}^{Y}+\beta_{i_{1} i_{2}}^{X_{1} X_{2}}+\beta_{i_{1} j}^{X_{1} Y}+\beta_{i_{2} j}^{X_{2} Y}+\beta_{i_{1} i_{2} j}^{X_{1} X_{2} Y} .
$$


O modelo correspondente no contexto do produto de multinomiais é escrito com base nos logitos e toma a forma:

$$
\ln \theta_{i_{1} i_{2}}=\lambda_{i_{1} i_{2}}+u_{i_{1} i_{2} j}^{Y}
$$

em que $\sum_{j} u_{i_{1} i_{2} j}^{Y}=0$.

Usualmente define-se $\lambda_{i_{1} i_{2}}=\frac{1}{J} \sum_{j} \ln \theta_{i_{1} i_{2} j}$ e $u_{i_{1} i_{2} j}^{Y}=\ln \theta_{i_{1} i_{2} j}-\lambda_{i_{1} i_{2}}$.

Considerando-se as seguintes transformações

$$
\begin{aligned}
\lambda_{i_{1} i_{2}} & =\beta_{0}+\beta_{i_{1}}^{X_{1}}+\beta_{i_{2}}^{X_{2}}+\beta_{i_{1} i_{2}}^{X_{1} X_{2}} \mathrm{e} \\
u_{i_{1} i_{2} j}^{Y} & =\beta_{j}^{Y}+\beta_{i_{1} j}^{X_{1} Y}+\beta_{i_{2} j}^{X_{2} Y}+\beta_{i_{1} i_{2} j}^{X_{1} X_{2} Y}
\end{aligned}
$$

o modelo pode ser escrito como no contexto multinomial.

Os estimadores de máxima verossimilhança dos parâmetros essenciais em $u_{i_{1} i_{2} j}^{Y}$ são os mesmos que os correspondentes no modelo log-linear no contexto multinomial.

Tanto a adoção do mecanismo de censura não-ignorável NMAR quanto a do mecanismo de censura ignorável MAR, acarretaria um modelo saturado, com o mesmo número de parâmetros (Baker e Laird (1988) e Park e Brown (1994)), implicando que não se tratam de modelos encaixados.

A Figura 3.4 mostra a evolução das freqüências esperadas sob o modelo NMAR, $m_{\mathbf{i} j k}$, em função do valor de $\Delta$, no caso do exemplo das eleições. Nota-se que quando $p$ se aproxima de 16 , o valor de $m_{\mathrm{i} j k}$ inicia tendência para uma situação de estabilidade.

As Figuras 3.5 e 3.6 mostram a evolução das freqüências esperadas sob os métodos de máxima verossimilhança e bayesiano. Como os valores de $v_{3}$ (última coluna da Tabela 3.10) estão fora do intervalo definido por $v_{1}$ e $v_{2}$, o método mais indicado é o bayesiano.

As freqüências esperadas obtidas a partir da imputação dos elementos com intenção de voto desconhecida, sob os mecanismos de censura não-ignorável e ignorável, estão apresentadas na Tabela 3.11. O Programa utilizado foi desenvolvido 


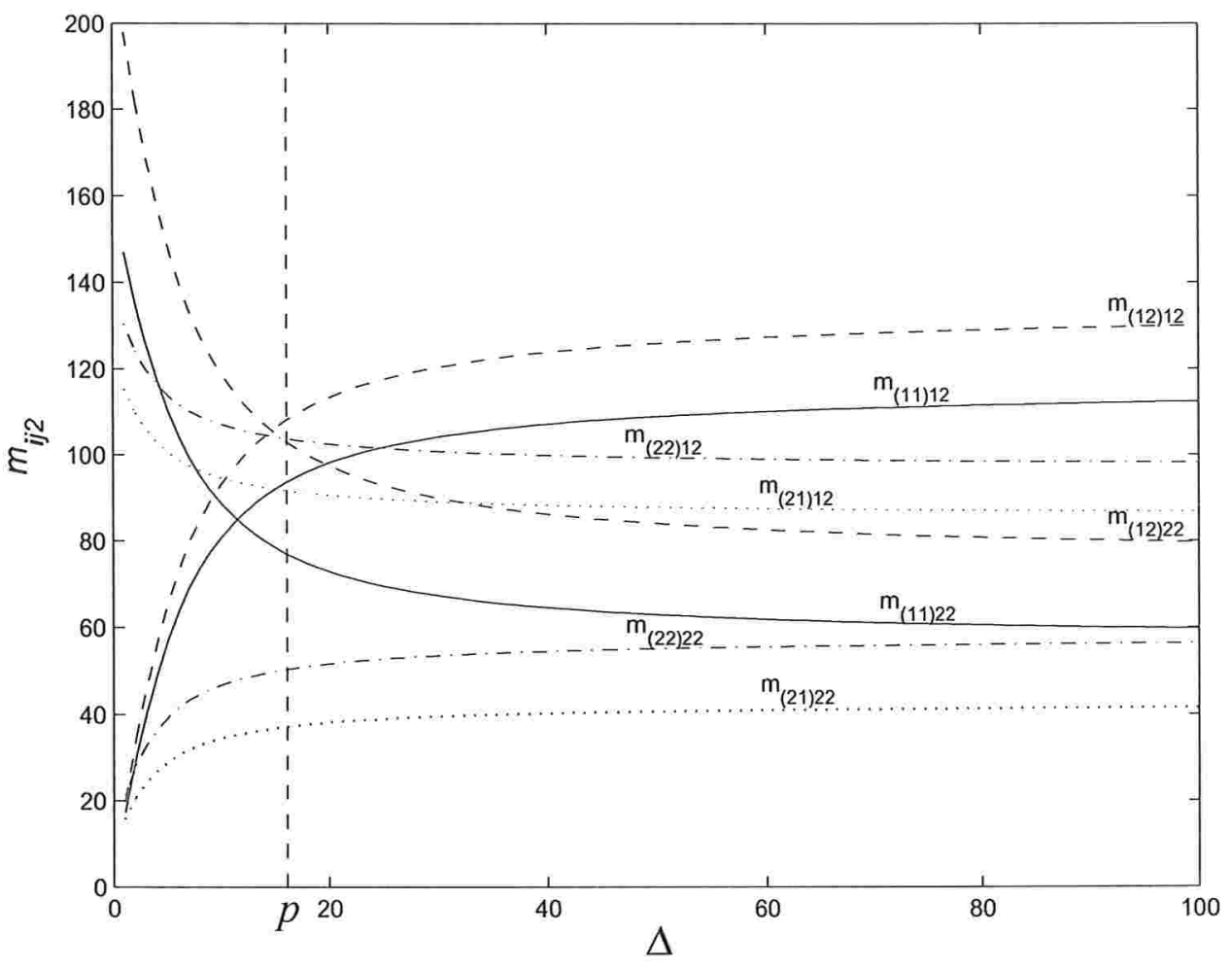

Figura 3.4: Estimativa de $m_{\mathbf{i} j k}$, eleições 2002, em função da escolha de $\Delta$.

para o aplicativo MatLab e encontra-se no Apêndice C. O algoritmo empregado no programa foi o IPF; entretanto, se o leitor preferir aplicar o algoritmo de Newton Raphson, as derivadas necessárias, para um caso simplificado, que podem ser generalizadas, também encontram-se nesse apêndice.

O resultado final das eleições para presidente 2002, oficialmente divulgado pela Justiça Eleitoral, dá ao candidato Lula $61,3 \%$ dos votos válidos e $38,7 \%$, ao candidato Serra. A Tabela 3.12 mostra os percentuais da preferência de voto sob os mecanismos de censura não-ignorável e ignorável primeiramente por período e idade, depois por período. O resultado da pesquisa de boca de urna divulgado pelo Datafolha e o resultado da eleição também são apresentados. Os resultados obtidos pelo modelo MAR são os mesmos divulgados pelo Datafolha, que consideram somente os dados totalmente observados. Nota-se claramente que o modelo NMAR é o que melhor se aproxima do resultado final das eleições, apresentando nos percentuais do 


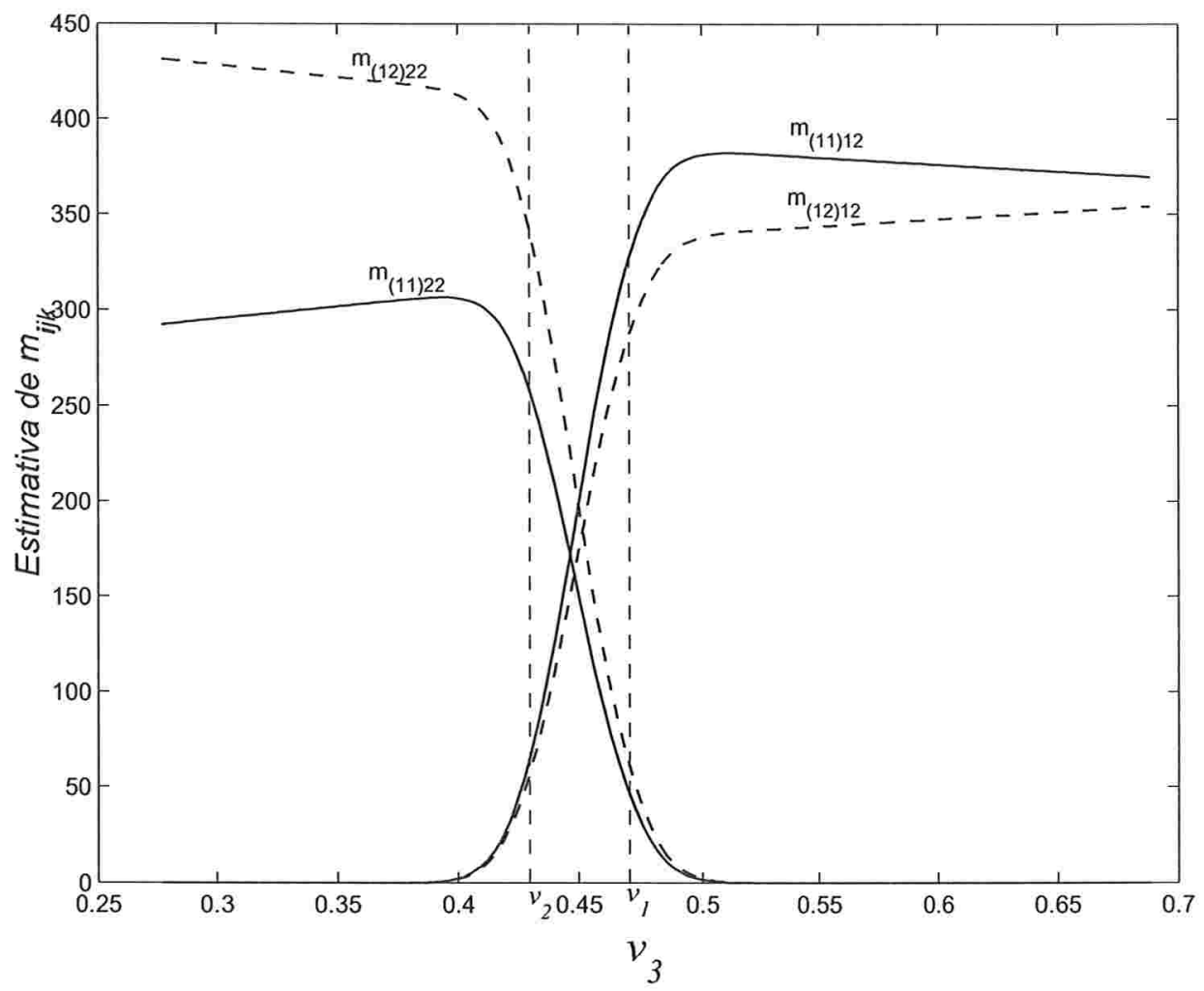

Figura 3.5: Evolução das freqüências esperadas sob o método da máxima verossimilhança, eleições 2002.

período $2(\mathrm{Lula}=61,1 \%$ e Serra $=38,9 \%)$ a sua melhor aproximação, sendo melhor até do que o resultado de boca de urna divulgado.

Embora as estimativas das proporções de votos tenham sido aqui apresentadas, intervalos de confiança não foram construídos pois o objetivo principal deste trabalho é a apresentação do método de imputação. A comparação desse tipo de método geralmente é feita de maneira empírica, mas aqui não fizemos comparação com outros métodos. 


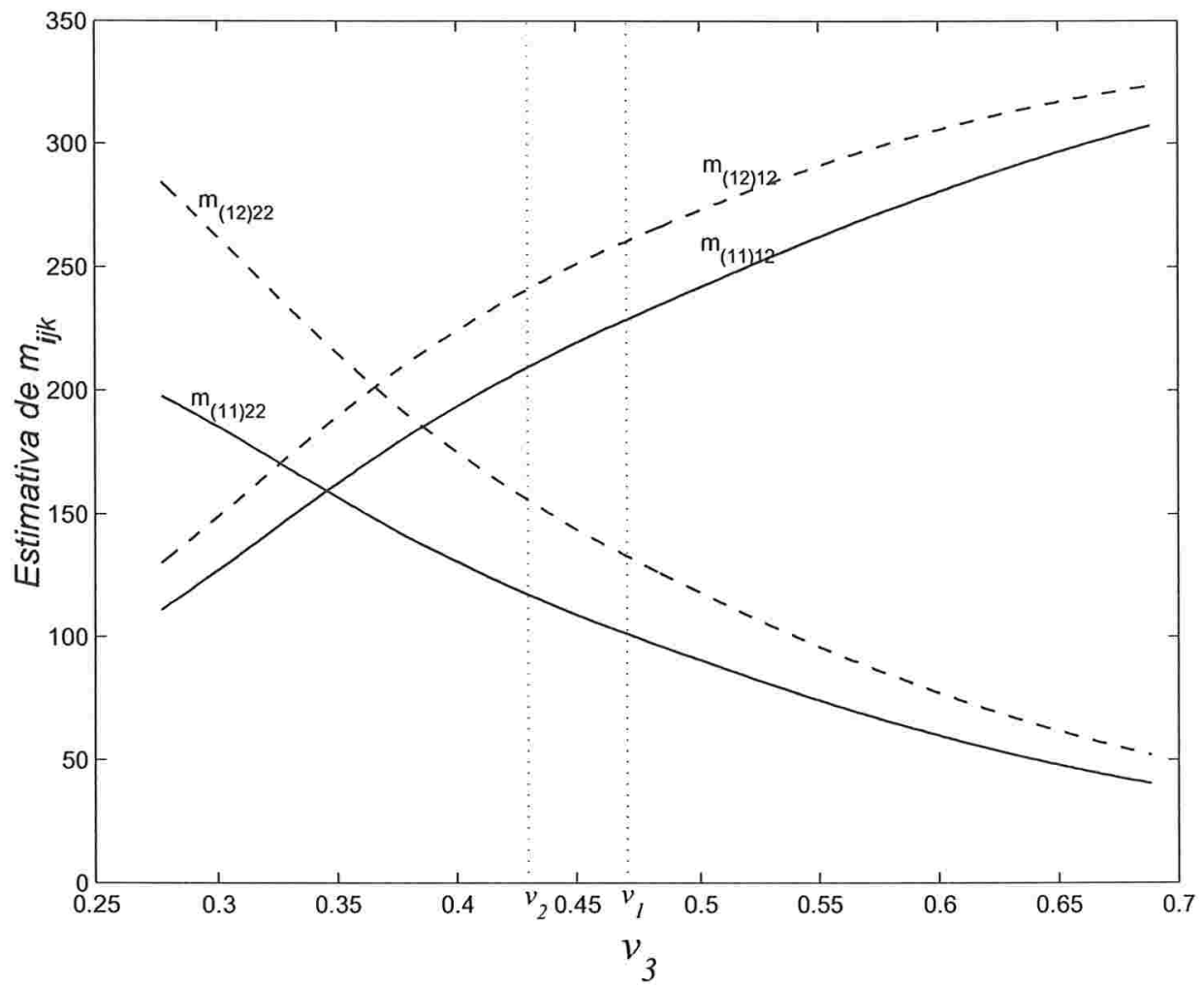

Figura 3.6: Evolução das frequiências esperadas sob o método bayesiano, eleições 2002.

Tabela 3.11: Intenções de voto com dados imputados - eleições 2002 - segundo turno

\begin{tabular}{cccccc}
\hline & & \multicolumn{3}{c}{ Intenção de Voto } \\
\cline { 3 - 6 } & & Não-Ignorável & \multicolumn{2}{c}{ Ignorável } \\
\cline { 3 - 6 } Período & Idade & Lula & Serra & Lula & Serra \\
\hline \multirow{2}{*}{1} & Até 34 anos & 3035 & 1598 & 3120 & 1513 \\
& Mais 34 anos & 3520 & 2244 & 3699 & 2065 \\
\hline \multirow{2}{*}{2} & Até 34 anos & 2952 & 1663 & 3078 & 1537 \\
& Mais 34 anos & 3393 & 2369 & 3605 & 2157 \\
\hline
\end{tabular}


Tabela 3.12: Percentuais na intenção de voto - eleições 2002 - segundo turno

\begin{tabular}{cccccc}
\hline & & \multicolumn{3}{c}{ Intenção de Voto } \\
\cline { 3 - 6 } & \multirow{2}{*}{ Período } & Idade & Não-Ignorável & \multicolumn{2}{c}{ Ignorável } \\
\cline { 3 - 6 } & Lula & Serra & Lula & Serra \\
\hline \multirow{2}{*}{2} & Até 34 anos & $65,5 \%$ & $34,5 \%$ & $67,3 \%$ & $32,7 \%$ \\
& Mais 34 anos & $61,1 \%$ & $38,9 \%$ & $64,2 \%$ & $35,8 \%$ \\
\hline \multirow{2}{*}{2} & Até 34 anos & $64,0 \%$ & $36,0 \%$ & $66,7 \%$ & $33,3 \%$ \\
& Mais 34 anos & $58,9 \%$ & $41,1 \%$ & $62,6 \%$ & $37,4 \%$ \\
\hline \multirow{2}{*}{2} & $63,0 \%$ & $37,0 \%$ & $65,6 \%$ & $34,4 \%$ \\
\hline \multirow{2}{*}{ Boca de urna } & $61,1 \%$ & $38,9 \%$ & $64,4 \%$ & $35,6 \%$ \\
\hline Resultado da eleição & $61,3 \%$ & $38,7 \%$ & & \\
\hline
\end{tabular}




\section{Capítulo 4}

\section{Conclusões}

Na Seção 3.1 apresentamos uma abordagem para o mecanismo de censura ignorável, juntamente com o problema das tabelas esparsas, vinculada ao exemplo do comsumo de drogas da Seção 2.1. A ocorrência dos dados esparsos mostrou-se um complicador na obtenção das estimativas dos parâmetros e dos erros-padrão além de colocar em dúvida a convergência assintótica da distribuição das estatísticas de Pearson e razão de verossimilhanças para uma distribuição $\chi^{2}$. Os procedimentos adotados para contornar esses problemas, ou seja, a adição de uma constante de ajuste e a simulação da distribuição da estatística do teste de ajuste, viabilizaram uma melhora considerável nos valores dos erros-padrão e no valor do teste para ajuste do modelo MCAR, que não foi rejeitado. Como a maioria dos casos reais, provavelmente o modelo NMAR seria mais adequado para este caso, entretanto, como comentado anteriormente, haveria grandes dificuldades para desenvolver um procedimento para obtenção das estimativas de interesse, dentro do contexto loglinear, em virtude no número de variáveis que deixam de ser observadas ser maior que um. Esse procedimento fica como sugestão para desenvolvimento em trabalho futuro.

A abordagem para o mecanismo de censura não-ignorável, apresentada na Seção 3.2, mostrou claramente a importância da escolha do mecanismo de censura que inclua as observações incompletas. A adoção de um modelo NMAR para o exem- 
plo das eleições da Seção 2.1 apresentou resultados mais próximos do valor final (votos válidos divulgados pela Justiça Eleitoral) se comparado com o resultado das pesquisas descartando-se os votos indefinidos ou adotando-se o modelo de censura ignorável.

Uma pergunta importante seria como eleger o modelo de censura adequado. Os modelos ignorável e não-ignorável não são encaixados; cada um deles se apresenta de forma independente para explicar os dados, ou seja, representam, individualmente, o modelo saturado. Provavelmente existem outros fatores que podem levar a uma escolha adequada do modelo de censura. Alguns desses fatores podem ser oferecidos pelo pesquisador, maior conhecedor do provável comportamento do fenômeno, outros por algum método de análise de sensibilidade. O desenvolvimento dessa análise de sensibilidade, será tema de um trabalho futuro. 


\section{Apêndice A}

\section{Estimação dos Parâmetros $\theta$ e $\lambda$.}

\section{Modelo de Censura Ignorável.}

Os passos do algoritmo EM são obtidos da seguinte forma:

Passo E:

$$
Q\left(\theta, \theta^{(g)}\right)=E\left\{\ln [p(\boldsymbol{\theta} \mid \mathbf{n})] \mid \mathbf{z}, \mathbf{u}, \theta^{(g)}\right\}
$$

onde podemos escrever,

$$
\begin{aligned}
\ln [p(\theta \mid \mathrm{n})]= & \ln N !+\sum_{i} \sum_{j} \sum_{k} n_{i j k} \ln \theta_{i j k} \\
& -\sum_{i} \sum_{j} \sum_{k} \ln n_{i j k} \\
\ln [p(\theta \mid \mathrm{n})] \propto & \sum_{i} \sum_{j} \sum_{k} n_{i j k} \ln \theta_{i j k} .
\end{aligned}
$$

Então,

$$
\begin{aligned}
& Q\left(\boldsymbol{\theta}, \boldsymbol{\theta}^{(g)}\right)=E\left\{\sum_{i} \sum_{j} \sum_{k} n_{i j k} \ln \theta_{i j k} \mid \mathbf{z}, \mathbf{u}, \theta^{(g)}\right\} \\
& Q\left(\boldsymbol{\theta}, \boldsymbol{\theta}^{(g)}\right)=\sum_{i} \sum_{j} \sum_{k} E\left\{n_{i j k} \mid \mathbf{z}, \mathbf{u}, \theta^{(g)}\right\} \ln \theta_{i j k} .
\end{aligned}
$$


Considerando-se o modelo multinomial da expressão (3.2), a função de verossimilhança pode ser escrita como

$$
L=\prod_{i j k}\left[\left(1-\lambda_{i j}^{12}-\lambda_{j k}^{13}-\lambda_{i}^{1}\right) \theta_{i j k}\right]^{z_{i j k}} \prod_{i j}\left(\lambda_{i j}^{12} \theta_{i j+}\right)^{u_{i j}^{12}} \prod_{i k}\left(\lambda_{i k}^{13} \theta_{i+k}\right)^{u_{i k}^{13}} \prod_{i}\left(\lambda_{i}^{1} \theta_{i++}\right)^{u_{i}^{1}}
$$

Pode-se fatorar a função de verossimilhança e escrevê-la como $L=L(\boldsymbol{\theta}) L(\boldsymbol{\lambda})$, em que

$$
L(\theta)=\prod_{i j k}\left(\theta_{i j k}\right)^{z_{i j k}} \prod_{i j}\left(\theta_{i j+}\right)^{u_{i j}^{12}} \prod_{i k}\left(\theta_{i+k}\right)^{u_{i k}^{13}} \prod_{i}\left(\theta_{i++}\right)^{u_{i}^{1}}
$$

e

$$
L(\lambda)=\prod_{i j k}\left(1-\lambda_{i j}^{12}-\lambda_{j k}^{13}-\lambda_{i}^{1}\right)^{z_{i j k}} \prod_{i j}\left(\lambda_{i j}^{12}\right)^{u_{i j}^{12}} \prod_{i k}\left(\lambda_{i k}^{13}\right)^{u_{i k}^{13}} \prod_{i}\left(\lambda_{i}^{1}\right)^{u_{i}^{1}}
$$

\section{Estimativa de $\theta$}

Para se determinar os estimadores de máxima verossimilhança de $\theta$ basta tomar $\ln L(\boldsymbol{\theta})$ e encontrar o valor de $\boldsymbol{\theta}$ que maximiza a expressão com a restrição de que a soma das probabilidades seja igual a 1 . Assim, fazendo-se uso dos multiplicadores de Lagrange, $\delta$, obtemos a equação de log-verossimilhança,

$$
\begin{aligned}
\ell(\boldsymbol{\theta})=\ln L(\boldsymbol{\theta}) & =\sum_{i j k} z_{i j k} \ln \theta_{i j k}+\sum_{i j} u_{i j}^{12} \ln \theta_{i j+}+\sum_{i k} u_{i k}^{13} \ln \theta_{i+k}+\sum_{i} u_{i}^{1} \ln \theta_{i++} \\
& -\delta\left[\left(\sum_{i j k} \theta_{i j k}\right)-1\right] .
\end{aligned}
$$

Logo,

$$
\begin{aligned}
&\left.\frac{\partial \ell(\boldsymbol{\theta})}{\partial \theta_{i j k}}\right|_{\substack{o_{i j k}=\hat{\theta}_{i j k} \\
\delta=\hat{\delta}}}=\frac{z_{i j k}}{\hat{\theta}_{i j k}}+\frac{u_{i j}^{12}}{\hat{\theta}_{i j+}}+\frac{u_{i k}^{13}}{\hat{\theta}_{i+k}}+\frac{u_{i}^{1}}{\hat{\theta}_{i++}}-\hat{\delta}=0 \\
& \hat{\delta}=\frac{z_{i j k}}{\hat{\theta}_{i j k}}+\frac{u_{i j}^{12}}{\hat{\theta}_{i j+}}+\frac{u_{i k}^{13}}{\hat{\theta}_{i+k}}+\frac{u_{i}^{1}}{\hat{\theta}_{i++}} \\
& \hat{\delta} \hat{\theta}_{i j k}=z_{i j k}+\frac{u_{i j}^{12}}{\hat{\theta}_{i j+}} \hat{\theta}_{i j k}+\frac{u_{i k}^{13}}{\hat{\theta}_{i+k}} \hat{\theta}_{i j k}+\frac{u_{i}^{1}}{\hat{\theta}_{i++}} \hat{\theta}_{i j k}
\end{aligned}
$$

e assim,

$$
\underbrace{\hat{\delta} \hat{\theta}_{i j k}}_{1}=\sum_{i j k}\left[z_{i j k}+\frac{u_{i j}^{12}}{\hat{\theta}_{i j+}} \hat{\theta}_{i j k}+\frac{u_{i k}^{13}}{\hat{\theta}_{i+k}} \hat{\theta}_{i j k}+\frac{u_{i}^{1}}{\hat{\theta}_{i++}} \hat{\theta}_{i j k}\right]
$$




$$
\begin{aligned}
& \hat{\delta}=\sum_{i j k}\left[z_{i j k}+\frac{u_{i j}^{12}}{\hat{\theta}_{i j+}} \hat{\theta}_{i j k}+\frac{u_{i k}^{13}}{\hat{\theta}_{i+k}} \hat{\theta}_{i j k}+\frac{u_{i}^{1}}{\hat{\theta}_{i++}} \hat{\theta}_{i j k}\right] \\
& \hat{\delta}=\sum_{i j k} z_{i j k}+\sum_{i j} u_{i j}^{12}+\sum_{i k} u_{i k}^{13}+\sum_{i} u_{i}^{1},
\end{aligned}
$$

o que leva a concluir que $\hat{\delta}=N$ e assim escrever,

$$
\begin{aligned}
N \hat{\theta}_{i j k} & =z_{i j k}+\frac{u_{i j}^{12}}{\hat{\theta}_{i j+}} \hat{\theta}_{i j k}+\frac{u_{i k}^{13}}{\hat{\theta}_{i+k}} \hat{\theta}_{i j k}+\frac{u_{i}^{1}}{\hat{\theta}_{i++}} \hat{\theta}_{i j k} \\
\hat{n}_{i j k} & =z_{i j k}+\frac{u_{i j}^{12}}{\hat{\theta}_{i j+}} \hat{\theta}_{i j k}+\frac{u_{i k}^{13}}{\hat{\theta}_{i+k}} \hat{\theta}_{i j k}+\frac{u_{i}^{1}}{\hat{\theta}_{i++}} \hat{\theta}_{i j k} .
\end{aligned}
$$

A partir dessa expressão e igualando-se $E\left\{n_{i j k} \mid \mathbf{z}, \mathbf{u}, \theta^{(g)}\right\}$ a $\hat{n}_{i j k}^{(g)}$ pode-se obter o passo $E$,

$$
\hat{n}_{i j k}^{(g)}=z_{i j k}+\frac{u_{i j}^{12}}{\hat{\theta}_{i j+}^{(g)}} \hat{\theta}_{i j k}^{(g)}+\frac{u_{i k}^{13}}{\hat{\theta}_{i+k}^{(g)}} \hat{\theta}_{i j k}^{(g)}+\frac{u_{i}^{1}}{\hat{\theta}_{i++}^{(g)}} \hat{\theta}_{i j k}^{(g)}
$$

sendo $g$ o índice da iteração.

\section{Passo M:}

Deve-se encontrar o valor de $\boldsymbol{\theta}$ que maximize $Q\left(\boldsymbol{\theta}, \boldsymbol{\theta}^{(g)}\right)$ e, então, através do uso dos multiplicadores de Lagrange, $\delta$, temos:

$$
\begin{gathered}
Q\left(\boldsymbol{\theta}, \boldsymbol{\theta}^{(g)}, \delta\right)=\sum_{i j k \ldots t} E\left\{n_{i j k} \mid \mathbf{z}, \mathbf{u}, \boldsymbol{\theta}^{(g)}\right\} \ln \theta_{i j k}-\delta\left[\left(\sum_{i j k} \theta_{i j k}\right)-\mathbf{1}\right] \\
\left.\frac{\partial Q\left(\boldsymbol{\theta}, \boldsymbol{\theta}^{(g)}, \delta\right)}{\partial \theta_{i j k}}\right|_{\substack{\left.\theta_{i j k}=\hat{\theta}_{i j k} \\
\delta=\hat{\delta} \\
E n_{i j k} \mid \mathbf{m}, \mathbf{u}, \theta^{(g)}\right\}=\hat{\mathbf{n}}_{i j k}^{(g)}}}=\frac{\hat{n}_{i j k}}{\hat{\theta}_{i j k}}-\hat{\delta}=0 \Rightarrow \hat{\theta}_{i j k}=\frac{\hat{n}_{i j k}}{\hat{\delta}}
\end{gathered}
$$

e assim temos,

$$
\sum_{i j k} \hat{\theta}_{i j k}=\frac{\sum_{i j k} \hat{n}_{i j k}}{\hat{\delta}} \rightarrow 1=\frac{N}{\hat{\delta}} \Rightarrow \hat{\delta}=N
$$

e podemos escrever,

$$
\hat{\theta}_{i j k}^{(g+1)}=\frac{\hat{n}_{i j k}^{(g)}}{N}
$$


Assim no exemplo das drogas, apresentado na Seção 2.1, as equações de recorrência podem ser escritas, com base nas Tabelas 2.1 e 3.3, como:

$$
\begin{aligned}
& \hat{n}_{111}^{(g)}=z_{111}+u_{11}^{12} \frac{\hat{\theta}_{111}^{(g)}}{\hat{\theta}_{111}^{(g)}+\hat{\theta}_{112}^{(g)}}+u_{11}^{13} \frac{\hat{\theta}_{111}^{(g)}}{\hat{\theta}_{111}^{(g)}+\hat{\theta}_{121}^{(g)}}+u_{1}^{1} \frac{\hat{\theta}_{111}^{(g)}}{\hat{\theta}_{11}^{(g)}+\hat{\theta}_{112}^{(g)}+\hat{\theta}_{121}^{(g)}+\hat{\theta}_{122}^{(g)}} \\
& \hat{n}_{112}^{(g)}=z_{112}+u_{11}^{12} \frac{\hat{\theta}_{112}^{(g)}}{\hat{\theta}_{111}^{(g)}+\hat{\theta}_{112}^{(g)}}+u_{12}^{13} \frac{\hat{\theta}_{112}^{(g)}}{\hat{\theta}_{112}^{(g)}+\hat{\theta}_{122}^{(g)}}+u_{1}^{1} \frac{\hat{\theta}_{112}^{(g)}}{\hat{\theta}_{111}^{(g)}+\hat{\theta}_{112}^{(g)}+\hat{\theta}_{121}^{(g)}+\hat{\theta}_{122}^{(g)}} \\
& \hat{n}_{121}^{(g)}=z_{121}+u_{12}^{12} \frac{\hat{\theta}_{121}^{(g)}}{\hat{\theta}_{121}^{(g)}+\hat{\theta}_{122}^{(g)}}+u_{11}^{13} \frac{\hat{\theta}_{121}^{(g)}}{\hat{\theta}_{111}^{(g)}+\hat{\theta}_{121}^{(g)}}+u_{1}^{1} \frac{\hat{\theta}_{111}^{(g)}}{\hat{\theta}_{111}^{(g)}+\hat{\theta}_{112}^{(g)}+\hat{\theta}_{121}^{(g)}+\hat{\theta}_{122}^{(g)}} \\
& \hat{n}_{122}^{(g)}=z_{122}+u_{11}^{12} \frac{\hat{\theta}_{122}^{(g)}}{\hat{\theta}_{121}^{(g)}+\hat{\theta}_{122}^{(g)}}+u_{12}^{13} \frac{\hat{\theta}_{122}^{(g)}}{\hat{\theta}_{112}^{(g)}+\hat{\theta}_{122}^{(g)}}+u_{1}^{1} \frac{\hat{\theta}_{122}^{(g)}}{\hat{\theta}_{111}^{(g)}+\hat{\theta}_{112}^{(g)}+\hat{\theta}_{121}^{(g)}+\hat{\theta}_{122}^{(g)}} \\
& \hat{n}_{211}^{(g)}=z_{211}+u_{21}^{12} \frac{\hat{\theta}_{211}^{(g)}}{\hat{\theta}_{211}^{(g)}+\hat{\theta}_{212}^{(g)}}+u_{21}^{13} \frac{\hat{\theta}_{211}^{(g)}}{\hat{\theta}_{211}^{(g)}+\hat{\theta}_{221}^{(g)}}+u_{2}^{1} \frac{\hat{\theta}_{211}^{(g)}}{\hat{\theta}_{211}^{(g)}+\hat{\theta}_{212}^{(g)}+\hat{\theta}_{221}^{(g)}+\hat{\theta}_{222}^{(g)}} \\
& \hat{n}_{212}^{(g)}=z_{212}+u_{21}^{12} \frac{\hat{\theta}_{212}^{(g)}}{\hat{\theta}_{211}^{(g)}+\hat{\theta}_{212}^{(g)}}+u_{22}^{13} \frac{\hat{\theta}_{212}^{(g)}}{\hat{\theta}_{212}^{(g)}+\hat{\theta}_{222}^{(g)}}+u_{2}^{1} \frac{\hat{\theta}_{211}^{(g)}}{\hat{\theta}_{211}^{(g)}+\hat{\theta}_{212}^{(g)}+\hat{\theta}_{221}^{(g)}+\hat{\theta}_{222}^{(g)}} \\
& \hat{n}_{221}^{(g)}=z_{221}+u_{22}^{12} \frac{\hat{\theta}_{221}^{(g)}}{\hat{\theta}_{221}^{(g)}+\hat{\theta}_{222}^{(g)}}+u_{21}^{13} \frac{\hat{\theta}_{221}^{(g)}}{\hat{\theta}_{211}^{(g)}+\hat{\theta}_{221}^{(g)}}+u_{2}^{1} \frac{\hat{\theta}_{211}^{(g)}}{\hat{\theta}_{211}^{(g)}+\hat{\theta}_{212}^{(g)}+\hat{\theta}_{221}^{(g)}+\hat{\theta}_{222}^{(g)}} \\
& \hat{n}_{222}^{(g)}=z_{222}+u_{22}^{12} \frac{\hat{\theta}_{222}^{(g)}}{\hat{\theta}_{221}^{(g)}+\hat{\theta}_{222}^{(g)}}+u_{22}^{13} \frac{\hat{\theta}_{222}^{(g)}}{\hat{\theta}_{212}^{(g)}+\hat{\theta}_{222}^{(g)}}+u_{2}^{1} \frac{\hat{\theta}_{211}^{(g)}}{\hat{\theta}_{211}^{(g)}+\hat{\theta}_{212}^{(g)}+\hat{\theta}_{221}^{(g)}+\hat{\theta}_{222}^{(g)}}
\end{aligned}
$$

para o passo $E$ e,

$$
\begin{array}{lll}
\hat{\theta}_{111}^{(g+1)}=\frac{\hat{n}_{111}^{(g)}}{N}, & \hat{\theta}_{112}^{(g+1)}=\frac{\hat{n}_{112}^{(g)}}{N}, \quad \hat{\theta}_{121}^{(g+1)}=\frac{\hat{n}_{121}^{(g)}}{N}, \quad \hat{\theta}_{122}^{(g+1)}=\frac{\hat{n}_{122}^{(g)}}{N}, \\
\hat{\theta}_{211}^{(g+1)}=\frac{\hat{n}_{211}^{(g)}}{N}, \quad \hat{\theta}_{212}^{(g+1)}=\frac{\hat{n}_{212}^{(g)}}{N}, \quad \hat{\theta}_{221}^{(g+1)}=\frac{\hat{n}_{221}^{(g)}}{N}, \quad \hat{\theta}_{222}^{(g+1)}=\frac{\hat{n}_{222}^{(g)}}{N},
\end{array}
$$

para o passo $M$.

A partir das expressões obtidas para os passos EM, foi escrito o programa a seguir para os aplicativos R ou S-Plus. 


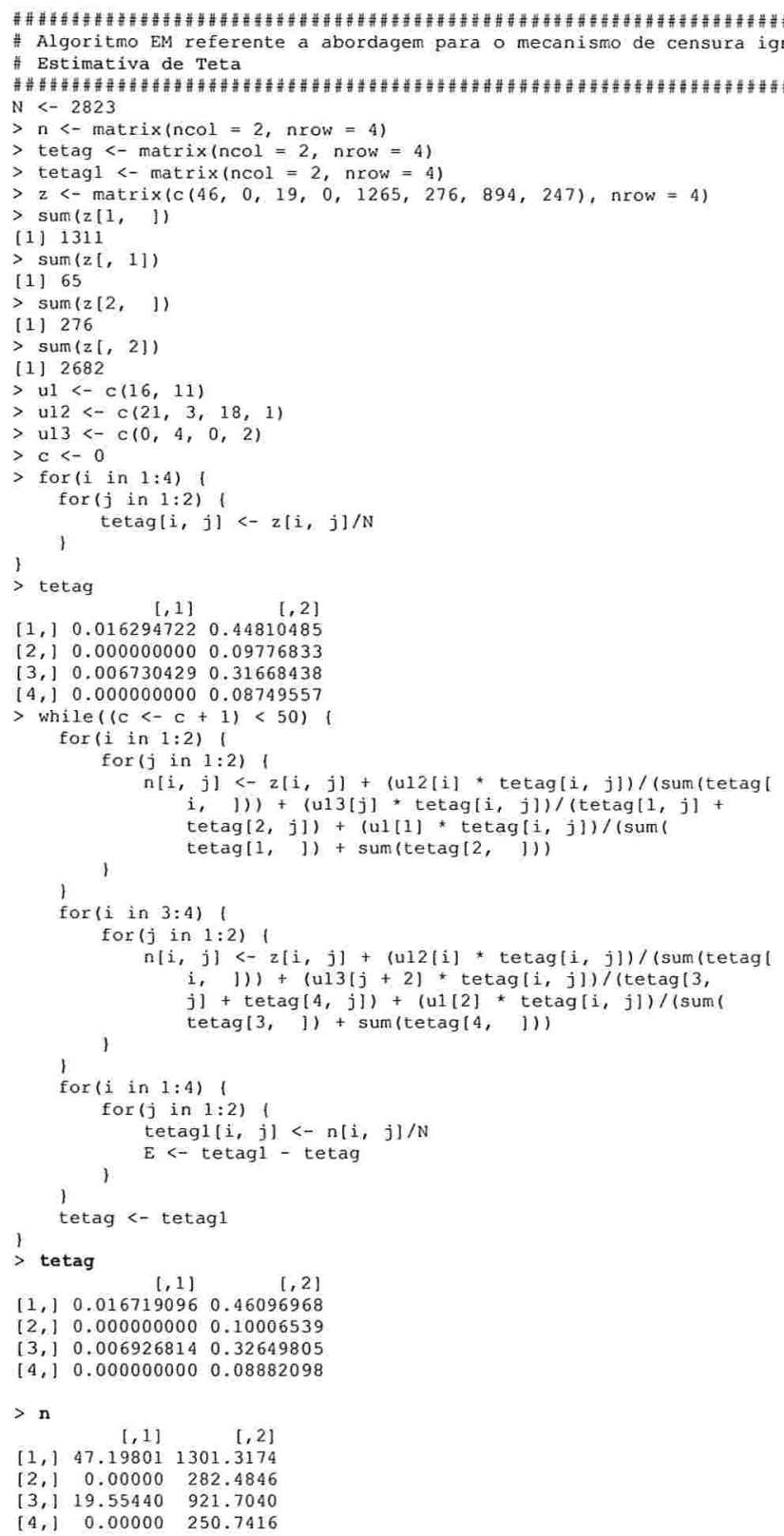




\section{Estimativa de $\lambda$}

Para determinação das estimativas de máxima verossimilhança (EMV's) das probabilidades de omissão de alguma(s) variável(is), $\lambda$, deve-se encontrar o valor representado por $\hat{\lambda}$, que maximiza a função $\ln L(\lambda)=\ell(\lambda)$.

$$
\begin{aligned}
\ell(\boldsymbol{\lambda}) & =\sum_{i j k} z_{i j k} \ln \left[1-\lambda_{i j}^{12}-\lambda_{i k}^{13}-\lambda_{i}^{1}\right]+\sum_{i j} u_{i j}^{12} \ln \lambda_{i j}^{12}+\sum_{i k} u_{i k}^{13} \ln \lambda_{i k}^{13} \\
& +\sum_{i} u_{i}^{1} \ln \lambda_{i}^{1}
\end{aligned}
$$

e assim,

$$
\frac{\partial \ell(\lambda)}{\partial \lambda}=\left[\begin{array}{c}
-\sum_{k}\left\{\frac{z_{i j k}}{1-\lambda_{i j}^{12}-\lambda_{i k}^{13}-\lambda_{i}^{1}}\right\}+\frac{u_{i j}^{12}}{\lambda_{i j}^{12}} \\
-\sum_{j}\left\{\frac{z_{i j k}}{1-\lambda_{i j}^{12}-\lambda_{i k}^{13}-\lambda_{i}^{1}}\right\}+\frac{u_{i k}^{13}}{\lambda_{i k}^{13}} \\
-\sum_{j} \sum_{k}\left\{\frac{z_{i j k}}{1-\lambda_{i j}^{12}-\lambda_{i k}^{13}-\lambda_{i}^{1}}\right\}+\frac{u_{i}^{1}}{\lambda_{i}^{1}}
\end{array}\right] .
$$

Assim, obtemos as seguintes expressões para os elementos de $\lambda$ :

$$
\begin{aligned}
\frac{u_{i j}^{12}}{\lambda_{i j}^{12}} & =\sum_{k}\left\{\frac{z_{i j k}}{1-\lambda_{i j}^{12}-\lambda_{i k}^{13}-\lambda_{i}^{1}}\right\}, \\
\frac{u_{i k}^{13}}{\lambda_{i k}^{13}} & =\sum_{j}\left\{\frac{z_{i j k}}{1-\lambda_{i j}^{12}-\lambda_{i k}^{13}-\lambda_{i}^{1}}\right\}, \\
\frac{u_{i}^{1}}{\lambda_{i}^{1}} & =\sum_{j} \sum_{k}\left\{\frac{z_{i j k}}{1-\lambda_{i j}^{12}-\lambda_{i k}^{13}-\lambda_{i}^{1}}\right\} .
\end{aligned}
$$

Essas expressões compõem um sistema de equações não-lineares cujas soluções são os EMV's dos componentes de $\lambda$. Para o exemplo das drogas esse sistema pode ser escrito como:

$$
\begin{aligned}
& \frac{u_{11}^{12}}{\hat{\lambda}_{11}^{12}}=\frac{z_{111}}{\left(1-\hat{\lambda}_{11}^{12}-\hat{\lambda}_{11}^{13}-\hat{\lambda}_{1}^{1}\right)}+\frac{z_{112}}{\left(1-\hat{\lambda}_{11}^{12}-\hat{\lambda}_{12}^{13}-\hat{\lambda}_{1}^{1}\right)}, \\
& \frac{u_{12}^{12}}{\hat{\lambda}_{12}^{12}}=\frac{z_{121}}{\left(1-\hat{\lambda}_{12}^{12}-\hat{\lambda}_{11}^{13}-\hat{\lambda}_{1}^{1}\right)}+\frac{z_{122}}{\left(1-\hat{\lambda}_{12}^{12}-\hat{\lambda}_{12}^{13}-\hat{\lambda}_{1}^{1}\right)}, \\
& \frac{u_{11}^{13}}{\hat{\lambda}_{11}^{13}}=\frac{z_{111}}{\left(1-\hat{\lambda}_{11}^{12}-\hat{\lambda}_{11}^{13}-\hat{\lambda}_{1}^{1}\right)}+\frac{z_{121}}{\left(1-\hat{\lambda}_{12}^{12}-\hat{\lambda}_{11}^{13}-\hat{\lambda}_{1}^{1}\right)}, \\
& \frac{u_{12}^{13}}{\hat{\lambda}_{12}^{13}}=\frac{z_{112}}{\left(1-\hat{\lambda}_{11}^{12}-\hat{\lambda}_{12}^{13}-\hat{\lambda}_{1}^{1}\right)}+\frac{z_{122}}{\left(1-\hat{\lambda}_{12}^{12}-\hat{\lambda}_{12}^{13}-\hat{\lambda}_{1}^{1}\right)},
\end{aligned}
$$




$$
\begin{aligned}
& \frac{u_{1}^{1}}{\hat{\lambda}_{1}^{1}}=\frac{z_{111}}{\left(1-\hat{\lambda}_{11}^{12}-\hat{\lambda}_{11}^{13}-\hat{\lambda}_{1}^{1}\right)}+\frac{z_{112}}{\left(1-\hat{\lambda}_{11}^{12}-\hat{\lambda}_{12}^{13}-\hat{\lambda}_{1}^{1}\right)}+\frac{z_{121}}{\left(1-\hat{\lambda}_{12}^{12}-\hat{\lambda}_{11}^{13}-\hat{\lambda}_{1}^{1}\right)}+\frac{z_{122}}{\left(1-\hat{\lambda}_{12}^{12}-\hat{\lambda}_{12}^{13}-\hat{\lambda}_{1}^{1}\right)}, \\
& \frac{u_{21}^{12}}{\hat{\lambda}_{21}^{12}}=\frac{z_{211}}{\left(1-\hat{\lambda}_{21}^{12}-\hat{\lambda}_{21}^{13}-\hat{\lambda}_{2}^{1}\right)}+\frac{z_{212}}{\left(1-\hat{\lambda}_{21}^{12}-\hat{\lambda}_{22}^{13}-\hat{\lambda}_{2}^{1}\right)}, \\
& \frac{u_{22}^{12}}{\hat{\lambda}_{22}^{12}}=\frac{z_{221}}{\left(1-\hat{\lambda}_{22}^{12}-\hat{\lambda}_{21}^{13}-\hat{\lambda}_{2}^{1}\right)}+\frac{z_{222}}{\left(1-\hat{\lambda}_{22}^{12}-\hat{\lambda}_{22}^{13}-\hat{\lambda}_{2}^{1}\right)}, \\
& \frac{u_{21}^{13}}{\hat{\lambda}_{21}^{13}}=\frac{z_{211}}{\left(1-\hat{\lambda}_{21}^{12}-\hat{\lambda}_{21}^{13}-\hat{\lambda}_{2}^{1}\right)}+\frac{z_{221}}{\left(1-\hat{\lambda}_{22}^{12}-\hat{\lambda}_{21}^{13}-\hat{\lambda}_{2}^{1}\right)}, \\
& \frac{u_{22}^{13}}{\hat{\lambda}_{22}^{13}}= \frac{z_{212}}{\left(1-\hat{\lambda}_{21}^{12}-\hat{\lambda}_{22}^{13}-\hat{\lambda}_{2}^{1}\right)}+\frac{z_{222}}{\left(1-\hat{\lambda}_{22}^{12}-\hat{\lambda}_{22}^{13}-\hat{\lambda}_{2}^{1}\right)}, \\
& \frac{u_{2}^{1}}{\hat{\lambda}_{2}^{1}}=\frac{z_{211}}{\left(1-\hat{\lambda}_{21}^{12}-\hat{\lambda}_{21}^{13}-\hat{\lambda}_{2}^{1}\right)}+\frac{z_{212}}{\left(1-\hat{\lambda}_{21}^{12}-\hat{\lambda}_{22}^{13}-\hat{\lambda}_{2}^{1}\right)}+\frac{z_{221}}{\left(1-\hat{\lambda}_{22}^{12}-\hat{\lambda}_{21}^{13}-\hat{\lambda}_{2}^{1}\right)}+\frac{z_{222}}{\left(1-\hat{\lambda}_{22}^{12}-\hat{\lambda}_{22}^{13}-\hat{\lambda}_{2}^{1}\right)} .
\end{aligned}
$$

O programa a seguir foi elaborado para o aplicativo Mathematica e corresponde ao método de Newton-Raphson aplicado ao exemplo das drogas.

Os parâmetros que compõem o vetor $\lambda=\left(\begin{array}{llllllllll}\lambda_{1}^{1} & \lambda_{11}^{12} & \lambda_{12}^{12} & \lambda_{11}^{13} & \lambda_{12}^{13} & \lambda_{21}^{12} & \lambda_{22}^{12} & \lambda_{21}^{13} & \lambda_{22}^{13} & \lambda_{2}^{1}\end{array}\right)^{\top}$ são representados, dentro da mesma seqüência, no programa por

$L 5, \quad L 1, \quad L 2, \quad L 3, L 4, L 6, L 7, \quad L 8, \quad L 9$ е $L 0$. 
Estimativas de Lambda

$\operatorname{In}(11]=$ FindRoot $[\{21.32 / \mathrm{L} 1=(46.32 /(1-\mathrm{L} 1-\mathrm{L} 3-\mathrm{I} 5))+(1265.32 /(1-\mathrm{L} 1-\mathrm{L} A-\mathrm{L} 5))$,

$3.32 / \mathrm{I} 2=(0.32 /(1-\mathrm{I} 2-\mathrm{I} 3-\mathrm{I} 5))+(276.32 /(1-\mathrm{I} 2-\mathrm{I} 4-\mathrm{IL}))$,

$0.32 / \mathrm{L} 3=(46.32 /(1-\mathrm{I} 1-\mathrm{L} 3-\mathrm{I} .5))+(0.32 /(1-\mathrm{L} 2-\mathrm{I} 3-\mathrm{L} 5))$,

$4.32 / L A==(1265.32 /(1-L 1-I A-L 5))+(276.32 /(1-I 2-L A-L 5))$,

$16.32 / \mathrm{L} 5=(46.32 /(1-\mathrm{L} 11-\mathrm{L} 3-\mathrm{L} 5))+(1265.32 /(1-\mathrm{L} 1-\mathrm{I} A-\mathrm{L} 5))+(0.32 /(1-\mathrm{L} 2-\mathrm{L} 3-\mathrm{IL} 5))+(276.32 /(1-\mathrm{L} 2-\mathrm{LA}-\mathrm{L} 5))$,

$18.32 / L 6=(19.32 /(1-L 6-I 8-L O))+(894.32 /(1-L 6-I 9-L O))$,

$1.32 / L 7=(0.32 /(1-L 7-I 8-L O))+(247.32 /(1-L 7-L 9-L O))$,

$0.32 / L 8=(19.32 /(1-L 6-I B-L 0))+(0.32 /(1-L 7-L B-L O))$,

$2.32 / 19=(894.32 /(1-L 6-L 9-10))+(247.32$

$11.32 / I D=(19.32 /(1-\mathrm{L} 6-\mathrm{I} 8-\mathrm{IO}))+(894.32 /(1-\mathrm{L} 6-\mathrm{L} . \mathrm{L}-\mathrm{LO}))+(0.32 /(1-\mathrm{L} 7-\mathrm{L} 8-\mathrm{L} 0))+(247.32 /(1-\mathrm{L} 7-\mathrm{L} .9-\mathrm{L} 0))\}$,

\{L1, .001\},

\{L2, .001\},

$\{$ [3, .001\},

$\{\mathrm{L}, .001\}$,

$\{$ L5, .001\},

$\{$ L6, .001\},

$\{$ L7, .001\},

$\{18, .001\}$,

(L9, .001),

(L, .001)

$\mathrm{Q} t[11]=(\mathrm{L} 1 \rightarrow 0.0155556, \mathrm{~L} 2 \rightarrow 0.011708, \mathrm{~L} 3 \rightarrow 0.00664041, \mathrm{~L} 4 \rightarrow 0.00272492, \mathrm{~L} 5 \rightarrow 0.00999045$,

$\mathrm{L} 6 \rightarrow 0.0190939, \mathrm{~L} 7 \rightarrow 0.00524124, \mathrm{~J} 8 \rightarrow 0.0155776, \mathrm{~L} 9 \rightarrow 0.00197612, \mathrm{~L} 0 \rightarrow 0.00947629$ \} 


\section{Apêndice B}

\section{Variâncias de $\hat{\theta}$ e $\hat{\lambda}$. Modelo de}

\section{Censura Ignorável.}

\section{Variância de $\hat{\theta}$}

Conforme resultados do Apêndice $\mathrm{A}$, a função de verossimilhança para $\theta$ é

$$
\ell(\theta)=\ln L(\theta)=\sum_{i j k} z_{i j k} \ln \theta_{i j k}+\sum_{i j} u_{i j}^{12} \ln \theta_{i j+}+\sum_{i k} u_{i k}^{13} \ln \theta_{i+k}+\sum_{i} u_{i}^{1} \ln \theta_{i++} .
$$

Assim, a função escore pode ser escrita como

$$
\frac{\partial \ell(\theta)}{\partial \theta_{i j k}}=\frac{z_{i j k}}{\theta_{i j k}}+\frac{u_{i j}^{12}}{\theta_{i j+}}+\frac{u_{i k}^{13}}{\theta_{i+k}}+\frac{u_{i}^{1}}{\theta_{i++}} .
$$

Os resultados de $\frac{\partial^{2} \ell(\boldsymbol{\theta})}{\partial \boldsymbol{\theta} \partial \boldsymbol{\theta}^{\top}}$ podem ser obtidos pela aplicação da função "Jacobiano" do aplicativo Matlab ao vetor escore e portanto é possível obter a matriz de covariância dada por $\operatorname{Var}(\hat{\boldsymbol{\theta}})=\left[-E\left(\frac{\partial^{2} \ell(\boldsymbol{\theta})}{\partial \boldsymbol{\theta} \partial \boldsymbol{\theta}}\right)\right]^{-1}$. Uma estimativa de $\operatorname{Var}(\hat{\boldsymbol{\theta}})$ é obtida pela substituição de $\theta$ por $\hat{\theta}$ nessa expressão, ou seja,

$$
\widehat{\operatorname{Var}}(\hat{\boldsymbol{\theta}})=\left[-E\left(\frac{\partial^{2} \ell(\boldsymbol{\theta})}{\partial \boldsymbol{\theta} \partial \boldsymbol{\theta}^{\top}}\right)\right]_{\hat{\boldsymbol{\theta}}}^{-1} .
$$

Usando-se o aplicativo MatLab, foi escrito o programa a seguir para se obter as estimativas dos erros-padrão de $\hat{\theta}$. A função escore foi substituída pelas funções $f 1$ a $f 8$ e, para os elementos do parâmetro $\theta$, foi utilizada a notação $t 1$ a $t 8$. Os 
valores estimados para os elementos do parâmetro $\theta$ foram obtidos com a adição da constante $\alpha=0,32$ nas celas. No caso em que essa adição não é necessária, o programa é similar.

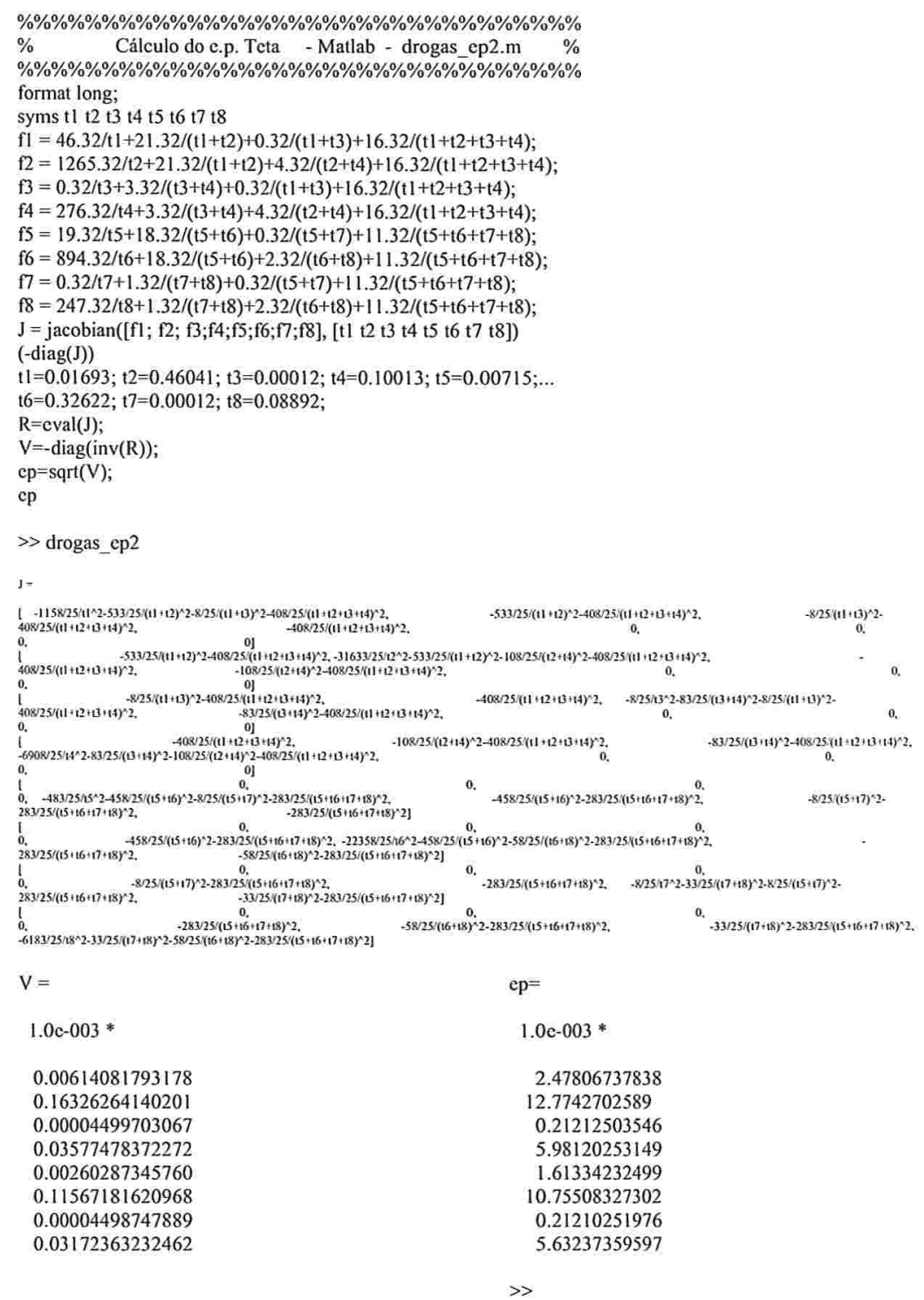




\section{Variância de $\hat{\lambda}$}

O vetor escore de $\lambda$, para o exemplo das drogas, pode ser escrito como

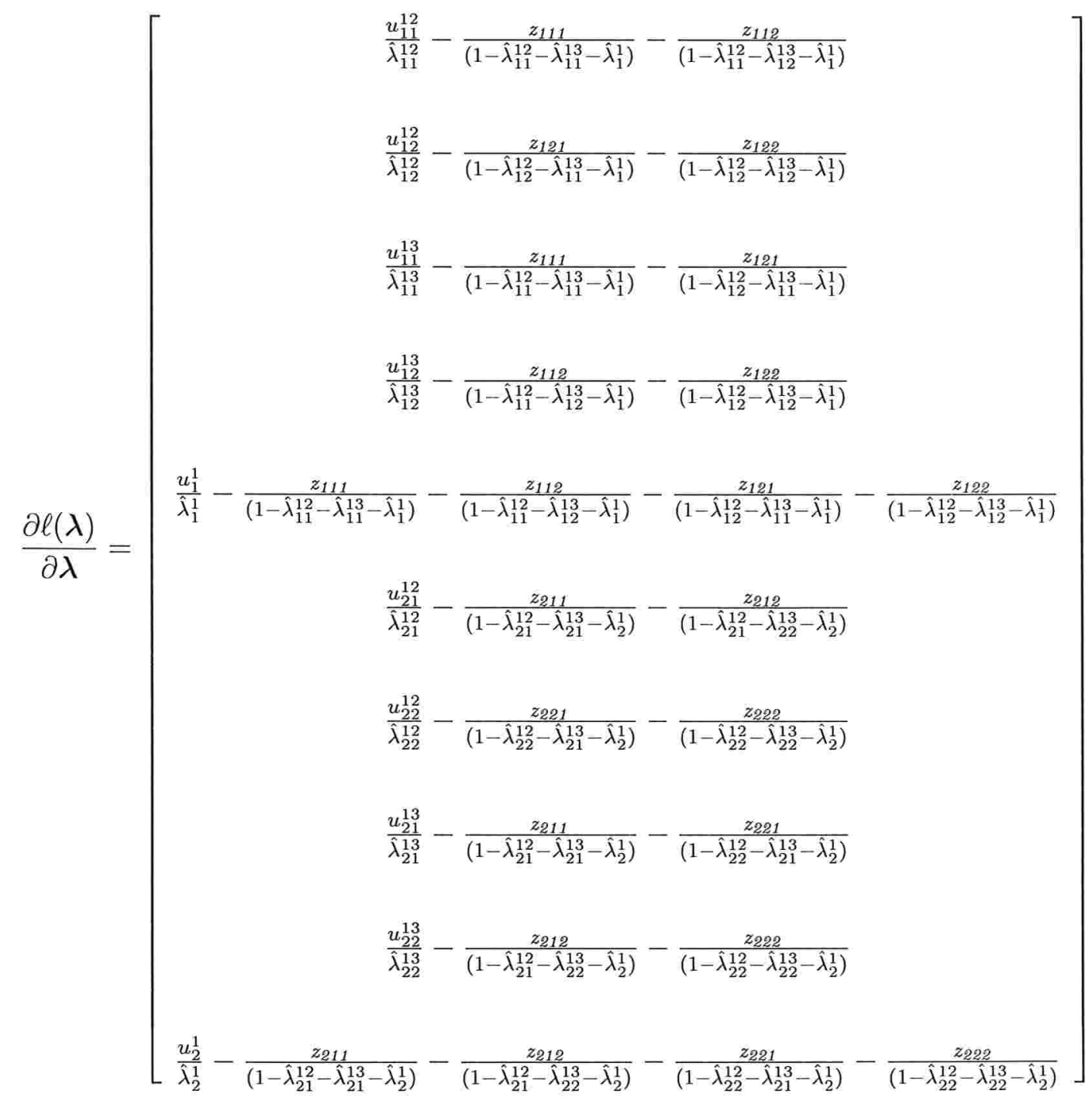

Aplicando-se novamente a função "Jacobiano" do aplicativo MatLab obtém-se o resultado de $\frac{\partial^{2} \ell(\boldsymbol{\lambda})}{\partial \boldsymbol{\lambda} \partial \boldsymbol{\lambda}^{\top}}$ e portanto é possível obter a matriz de covariância de $\hat{\boldsymbol{\lambda}}$ dada por $\operatorname{Var}(\hat{\boldsymbol{\lambda}})=\left[-E\left(\frac{\partial^{2} \ell(\boldsymbol{\lambda})}{\partial \boldsymbol{\lambda} \partial \boldsymbol{\lambda}}\right)\right]^{-1}$. A estimativa de $\operatorname{Var}(\hat{\boldsymbol{\lambda}})$ é obtida substituindo $\boldsymbol{\lambda}$ por $\hat{\lambda}$, ou seja,

$$
\widehat{\operatorname{Var}}(\hat{\lambda})=\left[-E\left(\frac{\partial^{2} \ell(\lambda)}{\partial \lambda \partial \lambda^{\top}}\right)\right]_{\hat{\lambda}}^{-1}
$$

Usando-se o aplicativo MatLab, foi escrito o programa a seguir para se obter as 
estimativas dos erros-padrão de $\hat{\lambda}$. Nesse programa a função escore foi substituída pelas funções $f 1$ a $f 10 \mathrm{e}$, para os elementos do parâmetro $\lambda$, foram utilizadas as denominações $L 1$ a $L 10$. Os valores estimados para o parâmetro $\boldsymbol{\lambda}$ foram obtidos com a adição da constante $\alpha=0,32$ nas celas. No caso em que essa adição não é necessária, o programa é similar. 


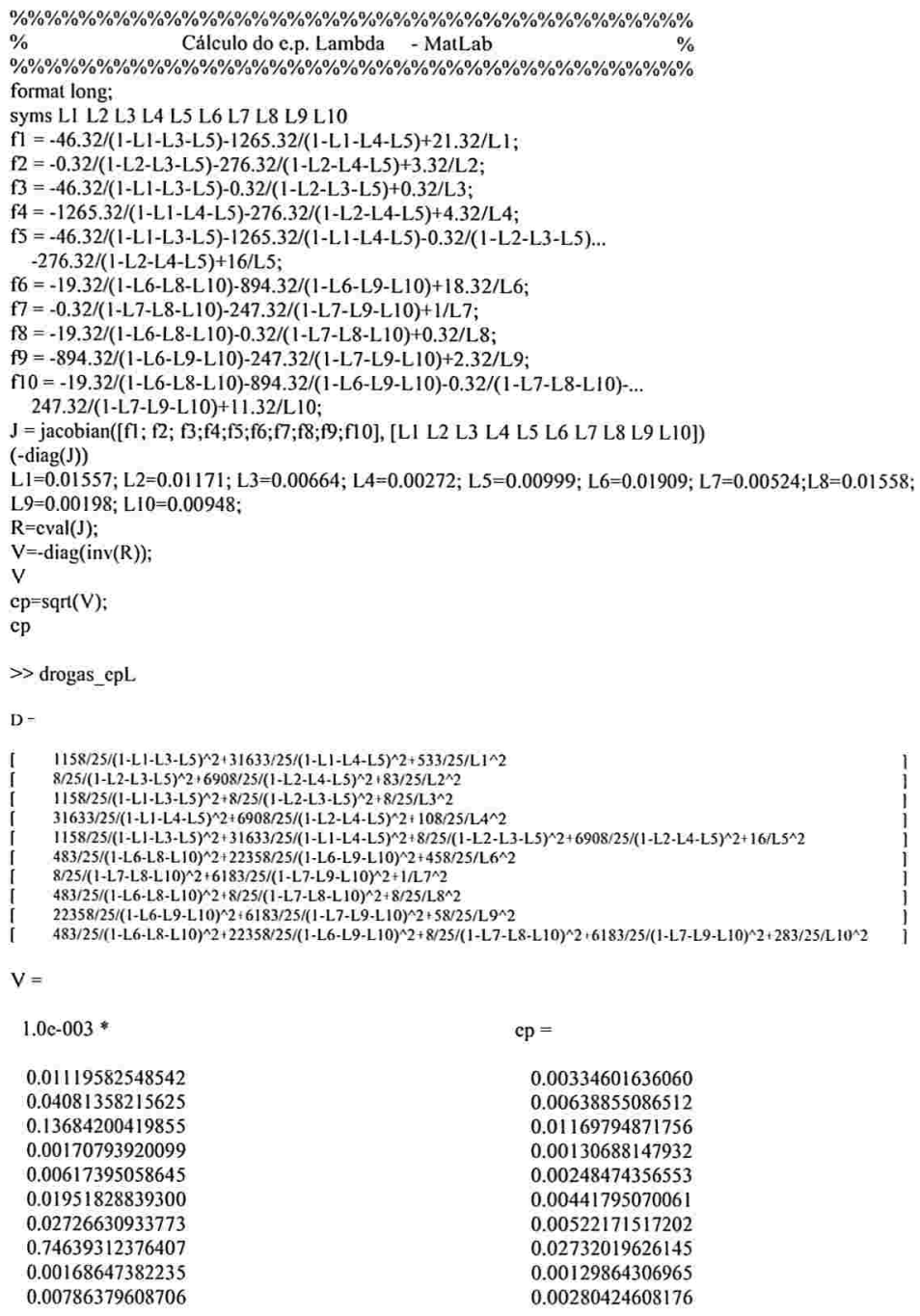




\section{Apêndice $\mathrm{C}$}

\section{Equações de Estimação para a}

\section{Aplicação 2}

Tomando-se a expressão (3.11),

$$
\begin{aligned}
p(\theta)= & \sum_{\mathbf{i}} \sum_{j} n_{\mathrm{i} j 1}\left[\ln \left(m_{\mathrm{i} j 1}\right)-\ln \left(m_{++1}\right)\right]+\sum_{\mathbf{i}}\left[n_{i+2}\left[\ln \left(m_{\mathbf{i}+2}\right)-\ln \left(m_{++2}\right)\right]+\right. \\
& \left.\sum_{j} \alpha_{\mathbf{i} j}\left[\ln \left(m_{\mathrm{i} j 2}\right)-\ln \left(m_{++2}\right)\right]\right]
\end{aligned}
$$

e a expressão para o modelo log-linear de censura não-ignorável (expressão (3.7)), vamos considerar que a variável Período seja fixada, isto é, os dados serão analisados para cada um dos dois períodos separadamente. Assim o vetor $\mathbf{X}$ fica reduzido a uma única variável, $X$, indexada por $i$.

Dessa forma, a Tabela 3.9 ficaria reduzida à Tabela C.1.

Tabela C.1: Freqüências esperadas com a introdução da variável $R$.

\begin{tabular}{cccc}
\hline & & \multicolumn{2}{c}{$R$} \\
\cline { 3 - 4 }$X$ & $\mathrm{Y}$ & $\operatorname{Sim}$ & Não \\
\hline 1 & 1 & $m_{111}$ & $m_{112}$ \\
& 2 & $m_{121}$ & $m_{122}$ \\
\cline { 2 - 4 } 2 & 2 & $m_{211}$ & $m_{212}$ \\
& 2 & $m_{221}$ & $m_{222}$ \\
\hline
\end{tabular}


A matriz de especificação $\mathrm{Z}$ é escrita como

$$
\mathrm{Z}=\left[\begin{array}{rrrrrrr}
1 & 1 & 1 & 1 & 1 & 1 & \rightarrow \mathrm{z}_{111} \\
1 & 1 & 1 & -1 & 1 & -1 & \rightarrow \mathrm{z}_{112} \\
1 & 1 & -1 & 1 & -1 & -1 & \rightarrow \mathrm{z}_{121} \\
1 & 1 & -1 & -1 & -1 & 1 & \rightarrow \mathrm{z}_{122} \\
1 & -1 & 1 & 1 & -1 & 1 & \rightarrow \mathrm{z}_{211} \\
1 & -1 & 1 & -1 & -1 & -1 & \rightarrow \mathrm{z}_{212} \\
1 & -1 & -1 & 1 & 1 & -1 & \rightarrow \mathrm{z}_{221} \\
1 & -1 & -1 & -1 & 1 & 1 & \rightarrow \mathrm{z}_{222}
\end{array}\right]
$$

Dentro do contexto do método de máxima verossimilhança temos que $\alpha_{i j}=0 \mathrm{e}$ as equações de verossimilhança podem ser escritas como

$$
\begin{aligned}
n_{i j 1}+n_{i j 2}^{*} & =m_{i j+} \quad \forall i, j=1,2, \\
n_{+j 1}=m_{+j 1} \text { e } n_{+j 2}^{*} & =m_{+j 2} \quad, \quad j=1,2 .
\end{aligned}
$$

Para o período 1, estas equações assumem a forma

$$
\begin{aligned}
& n_{111}+\left(n_{112}+n_{122}\right) \frac{m_{112}}{m_{112}+m_{122}}=m_{111}+m_{112} \\
& n_{121}+\left(n_{112}+n_{122}\right) \frac{m_{122}}{m_{112}+m_{122}}=m_{121}+m_{122} \\
& n_{211}+\left(n_{212}+n_{222}\right) \frac{m_{212}}{m_{212}+m_{222}}=m_{211}+m_{212} \\
& n_{221}+\left(n_{212}+n_{222}\right) \frac{m_{222}}{m_{212}+m_{222}}=m_{221}+m_{222} \\
& n_{111}+n_{211}=m_{111}+m_{211} \\
& n_{121}+n_{221}=m_{121}+m_{221} \\
& \left(n_{112}+n_{122}\right) \frac{m_{112}}{m_{112}+m_{122}}+\left(n_{212}+n_{222}\right) \frac{m_{212}}{m_{212}+m_{222}}=m_{112}+m_{212} \\
& \left(n_{112}+n_{122}\right) \frac{m_{122}}{m_{112}+m_{122}}+\left(n_{212}+n_{222}\right) \frac{m_{222}}{m_{212}+m_{222}}=m_{122}+m_{222}
\end{aligned}
$$

Essas equações correspondem ao passo $\mathrm{M}$ do algoritmo EM enquanto que o passo E é composto pelas expressões (3.15) e (3.16) tomando-se $\alpha_{i j}=0$.

É praticamente impossível obter uma solução para esse sistema de equações pelo método de Newton-Raphson, devido à extrema dificuldade na adoção do valor inicial 
do processo iterativo. Nesse sentido, surge a importância do uso do algoritmo EM, que tem a vantagem de convergir para uma solução mesmo se esta ocorrer nos limites do espaço paramétrico.

De qualquer forma, mesmo usando o algoritmo EM, a implementação computacional é extremamente trabalhosa o que abriria espaço para o uso do algoritmo IPF no passo $M$ tendo em vista maior simplicidade na implementação computacional com base nas freqüências marginais observadas.

Usando-se o aplicativo MatLab, foi escrito o programa a seguir para se obter as estimativas de $m_{\mathbf{i} j k}$ no ajuste do modelo não-ignorável para o exemplo das eleições com os dois períodos. 


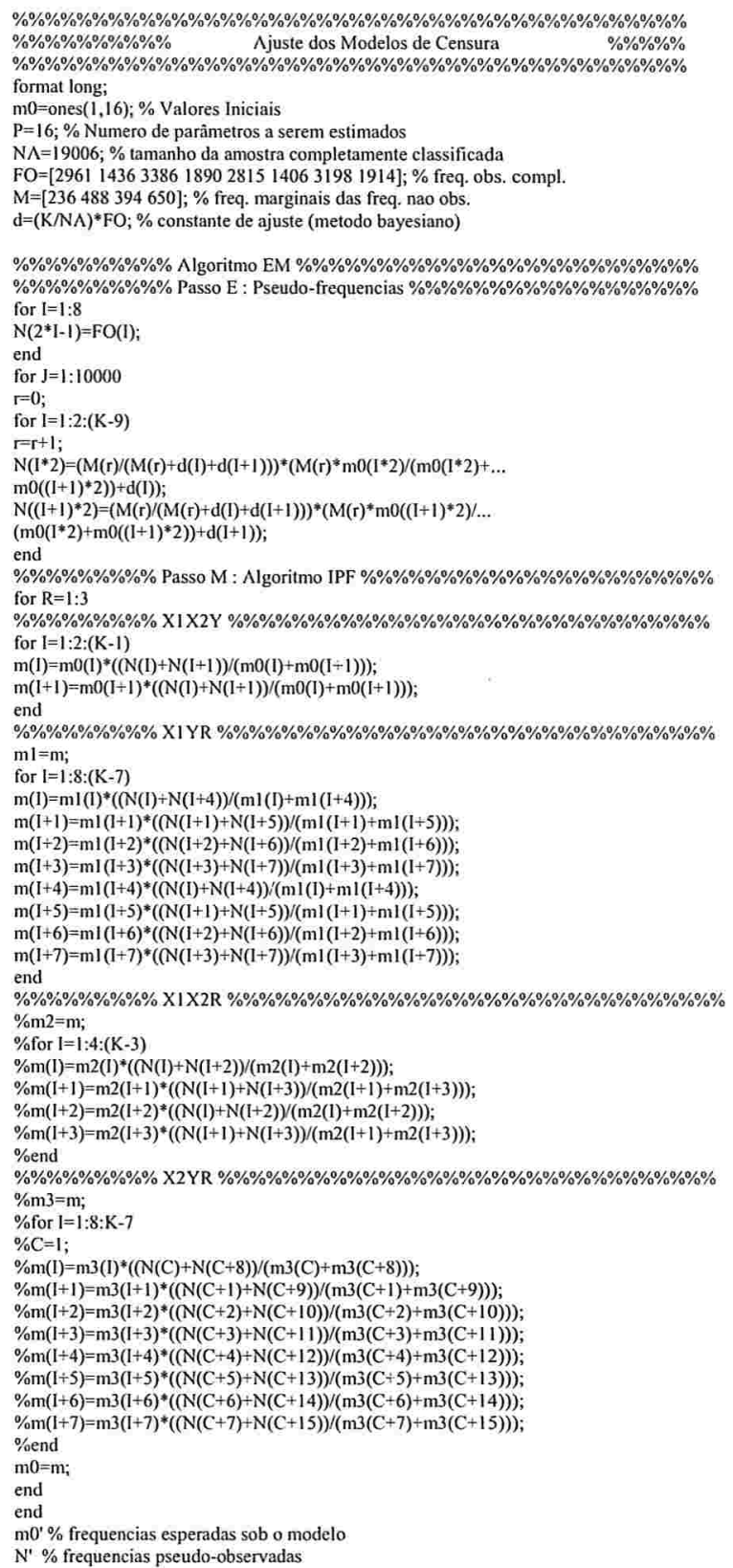


Abaixo temos os valores obtidos para as estimativas de $m_{\mathrm{i} j k}$.

$\gg$ ELEICOES_sat
ans $=$ (freqüências esper
$1.0 \mathrm{e}+003 *$
2.93910749455878
0.09638313597070
1.38300593693050
0.21450343254002
3.40789250544122
0.11175615976399
1.94299406306950
0.30135727172529
2.79743821536807
0.15469011884258
1.36939988415330
0.29347178163605
3.21556178463193
0.17781112443439
1.95060011584670
0.41802697508698

ans $=($ pseudo-frequêencias observadas $)$

$1.0 \mathrm{e}+003 *$

2.96100000000000

0.07449063052948

1.43600000000000

0.16150936947052

3.38600000000000

0.13364866520521

1.89000000000000

0.35435133479479

2.81500000000000

0.13712833421066

1.40600000000000

0.25687166578934

3.19800000000000

0.19537290906632

1.91400000000000

0.45462709093368 


\section{Apêndice D}

\section{Tópicos Utilizados}

Neste apêndice fazemos um resumo dos tópicos utilizados neste trabalho.

\section{D.1 Algoritmos}

\section{D.1.1 Algoritmo de Newton-Raphson}

Vamos supor que estejamos interessados em maximizar a função de log-verossimilhança $\ln L(\theta)=\ell(\theta)$ com respeito ao parâmetro $\theta=\left(\theta_{1}, \theta_{2}, \ldots, \theta_{p}\right)^{\top}$. Para cada passo do algoritmo de Newton-Raphson (representado por $g$ ), o valor atual estimado $\theta^{(g)}$ é atualizado como

$$
\boldsymbol{\theta}^{(g+1)}=\boldsymbol{\theta}^{(g)}+\left[-\frac{\partial^{2} \ell\left(\boldsymbol{\theta}^{(g)}\right)}{\partial \boldsymbol{\theta}^{(g)} \partial\left[\boldsymbol{\theta}^{(g)}\right]^{\top}}\right]^{-1} \frac{\partial \ell\left(\boldsymbol{\theta}^{(g)}\right)}{\partial \boldsymbol{\theta}^{(g)}} .
$$

Nessa expressão, $\frac{\partial^{2} \ell\left(\theta^{(g)}\right)}{\partial \boldsymbol{\theta}^{(g)} \partial\left[\boldsymbol{\theta}^{(g)}\right]^{\top}}$ corresponde à matriz das segundas derivadas, também chamada de matriz hessiana, com dimensão $p \times p$. Esse procedimento é repetido até a convergência, ou seja, até que $\boldsymbol{\theta}^{(g+1)}$ seja suficientemente próximo de $\boldsymbol{\theta}^{(g)}$.

O algoritmo de Newton-Raphson é obtido a partir de uma expansão de Taylor de primeira ordem da função escore em torno do valor $\theta^{(g)}$. Essa expansão caracteriza 
uma aproximação linear para a função escore e é da forma

$$
\frac{\partial \ell(\boldsymbol{\theta})}{\partial \boldsymbol{\theta}}=\frac{\partial \ell\left(\boldsymbol{\theta}^{(g)}\right)}{\partial \boldsymbol{\theta}^{(g)}}+\left(\boldsymbol{\theta}-\boldsymbol{\theta}^{(g)}\right) \frac{\partial^{2} \ell\left(\boldsymbol{\theta}^{(g)}\right)}{\partial \boldsymbol{\theta}^{(g)} \partial\left[\boldsymbol{\theta}^{(g)}\right]^{\top}}
$$

e assim, tomando-se a função escore linearizada igual a zero, obtém-se a equação (3.3).

A aproximação linear para a função escore implica que a função de log-verossimilhança deve ser aproximadamente quadrática. Quando a função de log-verossimilhança é exatamente quadrática então a função escore deverá ser exatamente linear e o algoritmo de Newton-Raphson convergirá no primeiro passo. Normalmente, a função de log-verossimilhança tende a tornar-se aproximadamente quadrática nas vizinhanças do ponto considerado quando o tamanho da amostra cresce. Quando o tamanho da amostra é demasiadamente pequeno a convergência pode ser prejudicada.

Uma vantagem do metódo de Newton-Raphson está no fato de produzir automaticamente, ao longo das iterações, a estimativa da matriz de covariância assintótica a partir da inversa da matriz hessiana, substituindo os parâmetros pelas suas estimativas naquele passo.

\section{D.1.2 Algoritmo EM}

A maximização da função de verossimilhança pode ser concretizada por métodos numéricos tais como Newton-Raphson ou "scoring" de Fisher, mas requerem geralmente um trabalho analítico pesado (MacLachlan e Krishnan, 1997). Além disso, a implementação destes métodos pode apresentar dificuldades numéricas (requisitos de memória computacional, falta de convergência, instabilidade, etc...), particularmente quando o número de parâmetros é grande. Uma alternativa para o método numérico direto de maximização da função de verossimilhança é conhecido como algoritmo de Esperança-Maximização ou EM (Dempster, Laird e Rubin, 1977). O algoritmo EM é um método geral de estimação de máxima verossimilhança para problemas com dados incompletos (MacLachlan e Krishnan, 1997). 
Vamos assumir um vetor aleatório $\mathbf{X}$ dividido em dois componentes, $\mathbf{X}_{o b s}$ e $\mathbf{X}_{p e r}$, correspondendo aos dados observados $\mathrm{x}_{\text {obs }}$ (completamente e incompletamente) e perdidos $\mathrm{x}_{\text {per }}$, respectivamente. Os dados observados podem ser representados como uma função dos dados completos, ou seja, $\mathbf{x}_{o b s}=h(\mathrm{x})$. A verossimilhança condicionada aos dados observados $\mathrm{x}_{o b s}$ é dada por

$$
L\left(\boldsymbol{\theta} \mid \mathrm{x}_{o b s}\right)=\int_{\aleph\left(\mathrm{x}_{o b s}\right)} L(\boldsymbol{\theta} \mid \mathbf{x}) d \mathbf{x},
$$

em que $\mathfrak{\aleph}\left(\mathrm{x}_{o b s}\right)$ corresponde ao espaço amostral, determinado por $\mathrm{x}_{o b s}=h(\mathbf{x})$ e $\theta=\left(\theta_{1}, \ldots, \theta_{p}\right)^{\top}$ é um vetor de parâmetros desconhecidos com espaço paramétrico $\Theta$.

Tomemos $p\left(\mathrm{x}_{\text {per }} \mid \mathrm{x}_{o b s}, \theta^{(g)}\right)$ como a distribuição condicional (aos dados observados e ao valor do parâmetro atual) preditora dos dados perdidos, $\mathbf{x}_{p e r}$.

Primeiramente, assume-se uma estimativa inicial $\left(\theta^{(0)}\right)$ para o parâmetro de interesse. Assim, na primeira iteração, o passo E requer

$$
Q\left(\boldsymbol{\theta}, \boldsymbol{\theta}^{(0)}\right)=E\left[\ln p(\boldsymbol{\theta} \mid \mathbf{x}) \mid \mathbf{x}_{o b s}, \boldsymbol{\theta}^{(0)}\right] .
$$

O passo M requer a maximização de $Q\left(\boldsymbol{\theta}, \boldsymbol{\theta}^{(0)}\right)$ com relação a $\boldsymbol{\theta}$ dentro do espaço paramétrico $\Theta$, isto é, escolhe-se $\theta^{(1)}$ de tal forma que

$$
Q\left(\boldsymbol{\theta}^{(1)}, \boldsymbol{\theta}^{(0)}\right) \geq Q\left(\boldsymbol{\theta}, \boldsymbol{\theta}^{(0)}\right)
$$

para todo $\theta \in \Theta$. Na iteração $(g+1)$, os passos EM ficam definidos como:

\section{Passo E}

Calcula-se

$$
\begin{aligned}
Q\left(\theta, \theta^{(g)}\right) & =\int_{\mathbf{x}_{p e r}} \ln \left[p\left(\theta \mid \mathbf{x}_{o b s}, \mathbf{x}_{p e r}\right)\right] p\left(\mathbf{x}_{p e r} \mid \boldsymbol{\theta}^{(g)}, \mathbf{x}_{o b s}\right) d \mathbf{x}_{p e r} \\
& =E\left[\ln p(\boldsymbol{\theta} \mid \mathbf{x}) \mid \mathbf{x}_{o b s}, \boldsymbol{\theta}^{(g)}\right]
\end{aligned}
$$

em que $\ln \left[p\left(\boldsymbol{\theta} \mid \mathbf{x}_{\text {obs }}, \mathbf{x}_{p e r}\right)\right]=\ln [p(\boldsymbol{\theta} \mid \mathbf{x})]$ é o logaritmo da função de densidade dos dados completos e $p\left(\mathrm{x}_{\text {per }} \mid \theta^{(g)}, \mathrm{x}_{\text {obs }}\right)$ é a função de densidade dos dados perdidos 
condicionados aos dados observados e ao valor atual do parâmetro de interesse para os dados completos.

\section{Passo M}

Encontra-se o valor de $\theta$ que maximiza $Q\left(\boldsymbol{\theta}, \boldsymbol{\theta}^{(g)}\right)$ de tal forma que

$$
Q\left(\theta^{(g+1)}, \theta^{(g)}\right) \geq Q\left(\theta, \theta^{(g)}\right)
$$

para todo $\theta \in \Theta$.

Itera-se até a convergência, ou seja, até que $\left|Q\left(\theta^{(g+1)}, \theta^{(g)}\right)-Q\left(\theta^{(g)}, \theta^{(g)}\right)\right|$ ou $\left\|\theta^{(g+1)}-\theta^{(g)}\right\|$ seja suficientemente pequeno.

\section{D.1.3 Algoritmo EM Generalizado}

Freqüentemente, na prática, existe uma solução fechada para a equação obtida no passo M. Nas situações onde isso não ocorre, pode não ser possível obter diretamente o valor de $\boldsymbol{\theta}$ que acarrete o máximo global da função $Q\left(\boldsymbol{\theta}, \boldsymbol{\theta}^{(g)}\right)$. Nesses casos, Dempster, Laird e Rubin (1977) definiram o algoritmo EM Generalizado (GEM no inglês) onde o passo $\mathrm{M}$ requer que $\theta^{(g+1)}$ seja escolhido de maneira que

$$
Q\left(\theta^{(g+1)}, \theta^{(g)}\right) \geq Q\left(\theta^{(g)}, \theta^{(g)}\right)
$$

Assim, escolhe-se $\theta^{(g+1)}$ para criar um incremento em $Q\left(\theta, \theta^{(g)}\right)$ sob o valor assumido por esta em $\theta=\theta^{(g)}$.

Outras formulações para o Algoritmo EM podem ser vistas em McLachlan e Krishnan (1997).

\section{D.1.4 Algoritmo IPF}

O método do ajustamento proporcional iterativo (do termo em inglês, Iterative Proportional Fitting), originalmente apresentado por Deming e Stephan (1940), é um procedimento que permite obter a estimativa de máxima verossimilhança de $\boldsymbol{\theta} \mathrm{com}$ base nas freqüências marginais observadas, as quais constituem estatísticas suficien- 
tes mínimas dentro do modelo log-linear reduzido enfocado. Para o modelo saturado, as estatísticas suficientes mínimas são as freqüências observadas em cada cela.

Sob determinadas condições, comparado com o método de Newton-Raphson, o algoritmo IPF tende a convergir mais lentamente; entretanto é computacionalmente mais simples. Ao longo do processo iterativo, o método de Newton-Raphson provê automaticamente a estimativa da matriz de covariância assintótica referente às estimativas dos parâmetros e o algoritmo IPF não fornece esta matriz.

O algoritmo IPF consiste das seguintes etapas :

1. uso de um valor inicial $\mathrm{m}^{(0)}$ que satisfaça o modelo log-linear a ajustar;

2. ajuste sucessivo dos elementos de $\mathbf{m}^{(0)}$ a cada um dos subconjuntos distintos de componentes da estatística suficiente mínima (freqüências marginais observadas), através de sua multiplicação por fatores de escala apropriados, de modo que satisfaça a correspondente equação de verossimilhança;

3. o processo continua até que a variação entre as estimativas seja desprezível dentro de um critério de convergência estabelecido a priori, o que acontece quando todas as equações de verossimilhança são satisfeitas dentro da aproximação tolerada.

Numa tabela $I \times J \times K$, para o modelo log-linear de ausência de interação de segunda ordem, o algoritmo IPF (adaptado às freqüências pseudo-observadas) é composto por três passos para cada iteração, pois nessa tabela temos três componentes da estatística suficiente mínima:

$$
\begin{aligned}
& m_{\mathbf{i} j k}^{(3 g+1)}=m_{\mathbf{i} j k}^{(3 g)} \frac{n_{\mathbf{i} j+}^{*}}{m_{\mathbf{i} j+}^{(3 g)}} \\
& m_{\mathbf{i} j k}^{(3 g+2)}=m_{\mathbf{i} j k}^{(3 g+1)} \frac{n_{\mathbf{i}+k}^{*}}{m_{\mathbf{i}+k}^{(3 g+1)}} \\
& m_{\mathbf{i} j k}^{(3 g+3)}=m_{\mathbf{i} j k}^{(3 g+2)} \frac{n_{+j k}^{*}}{m_{+j k}^{(3 g+2)}}
\end{aligned}
$$

Assim, $g$ corresponde ao número seqüencial da iteração e varia a cada três passos assumindo os valores $g=0,1,2, \ldots$ 


\section{D.2 Ajuste de Modelos}

Uma vez obtidos os estimadores dos parâmetros do modelo enfocado, é necessário verificar a qualidade do ajuste desse modelo restrito em contraposição com o modelo saturado representado pelas freqüências observadas. Basicamente, a verificação da qualidade do ajuste se dá pela confrontação entre os valores esperados sob o modelo restrito com os valores das freqüências observadas. As estatísticas mais usadas dentro desse contexto são:

\section{A Estatística da Razão de Verossimilhanças de Wilks}

Seguindo o contexto e a notação adotados em (3.1), essa estatística pode ser expressa por

$$
Q_{V}=-2 \sum_{i} \sum_{j} \sum_{k} n_{i j k \ldots t} \ln \left(\frac{m_{i j k}}{n_{i j k}}\right)
$$

em que $m_{i j k}$ é a freqüência esperada para a cela $i j k$ sob o modelo reduzido.

Tomando-se as hipóteses:

$H_{0}$ : modelo reduzido é adequado

$H_{A}$ : modelo reduzido não é adequado

temos então que, sob a hipótese nula, $Q_{V}$ converge assintoticamente para uma distribuição qui-quadrado quando o tamanho da amostra tende para o infinito. O número de graus de liberdade é dado pelo número de parâmetros sob $H_{A}$ menos o número de parâmetros sob a hipótese nula.

\section{A Estatística de Pearson}

A medida mais difundida da distância entre $\mathrm{m}$ (vetor coluna das freqüências esperadas sob o modelo reduzido) e $\mathbf{n}$ é dada por:

$$
Q_{P}=\sum_{i} \sum_{j} \sum_{k} \frac{\left(n_{i j k}-m_{i j k}\right)^{2}}{m_{i j k}}
$$


A estatística de Pearson $\left(Q_{P}\right)$ também converge, sob a hipótese nula, assintoticamente para uma distribuição qui-quadrado quando o tamanho da amostra tende para o infinito. O número de graus de liberdade é obtido de forma idêntica ao teste da razão de verossimilhanças.

\section{D.3 Dados Esparsos}

Uma tabela de contingência é esparsa quando possui várias celas com pequenas freqüências. Se $c$ é o número de celas e $N$ é o tamanho total da amostra, então $N / c$ é o indicador do quanto a tabela é esparsa, ou seja, quanto menor o indicador, mais esparsa é a tabela. Assim, podemos dizer que uma tabela é esparsa quando o tamanho da amostra é pequeno ou quando, sendo a amostra grande, existem muitas variáveis ou categorias, diluindo muito a amostra e gerando celas com pequenas freqüências. Mesmo para um número reduzido de variáveis e categorias, podem ocorrer eventos raros, como a Aplicação 1, em que as freqüências em determinadas celas são baixas, caracterizando uma tabela esparsa.

O uso da distribuição $\chi^{2}$ para o teste de ajuste é baseado em um resultado assintótico no qual cada freqüência esperada do modelo a ajustar seja suficientemente grande. Nesse sentido, a cela com menor valor esperado deve possuir esse mesmo valor convergindo para o infinito quando o tamanho da amostra convergir para o infinito.

Nessas condições, a grande questão é como decidir se o valor da cela é suficientemente grande e, conseqüentemente, se a condição de convergência assintótica para uma distribuição $\chi^{2}$ está sendo respeitada.

Com relação ao teste $\chi^{2}$ de Pearson, Cramér (1946) supõe que todos os valores das celas na tabela de contingência, sob o modelo reduzido, devem ser no mínimo 10. Fisher e Yates (1938) estabelecem que deveria ser pelo menos 5. Jeffreys (1961) sugere que 1 seria suficiente. Yarnold (1970) propôs que o valor mínimo esperado para uma cela deveria ser $b / c$, onde $c$ é o número total de celas e $b$ o número de 
celas onde o valor esperado é inferior a 5 . Cochran $(1942,1954)$ recomenda uma regra mais geral assumindo que um valor esperado mínimo de 1 é permissível desde que não mais de $20 \%$ dos valores esperados nas celas sejam menores que 5 . Caso contrário, é questionável que a distribuição $\chi^{2}$ sirva como referência.

$\mathrm{Na}$ prática, os estatísticos costumam usar o rótulo "esparsa" para caracterizar as tabelas de contingência que possuam celas com freqüências esperadas inferiores a cinco. De qualquer forma, essa condição está longe de ser conservadora e não oferece segurança absoluta na comparação de modelos. Como o modelo assumido introduz restrições nas proporções em cada cela, o número de parâmetros livres a serem estimados é freqüentemente menor que o número de celas. É interessante observar que, num modelo como o log-linear, nem sempre é necessário estimar as proporções nas celas com base na freqüência observada em cada uma delas. Assim, o entendimento de que determinada tabela é esparsa deve variar conforme o contexto do modelo proposto. Se levarmos em conta a redução de parâmetros, uma tabela pode ser considerada esparsa para determinado modelo mais complexo (maior número de parâmetros a serem estimados) e não ser para um modelo mais simples (menor número de parâmetros).

Celas vazias e tabelas esparsas podem causar inconvenientes na obtenção das estimativas dos parâmetros de um modelo log-linear. Podem não permitir a obtenção das estimativas. Podem causar sério viés nas medidas usualmente utilizadas em dados categorizados, por exemplo, a razão de chances. Também podem afetar o erro-padrão desses estimadores, além dos problemas envolvendo a performance de algoritmos computacionalmente implementados e da aproximação assintótica pela distribuição $\chi^{2}$.

Segundo Larntz (1978), Koehler e Larntz (1980) e Koehler (1986) o teste de Pearson baseado em $Q_{P}$ é mais representativo do que o teste da razão de verossimilhanças de Wilks baseado em $Q_{V}$ (levando-se em conta uma aproximação pela distribuição $\chi^{2}$ ) quando se tem uma tabela esparsa, ou seja, o teste baseado em $Q_{P}$ apresenta melhor aproximação pela distribuição $\chi^{2}$ quando $N / c<5$. Quando 
a maior parte das freqüências esperadas são menores do que 0,5 , a aproximação da distribuição de $Q_{V}$ pela distribuição de $\chi^{2}$ é conservadora, ou seja, o p-valor é maior do que realmente deveria ser. Quando a maioria das freqüências esperadas se encontra entre 0,5 e 4,0, o teste baseado em $Q_{V}$ apresenta um valor subestimado para o p-valor (é menor do que realmente deveria ser).

Numa situação em que a tabela é considerada esparsa, sendo pouco prudente usar uma aproximação pela $\chi^{2}$ para os testes de ajuste, é necessário encontrar algum procedimento para contornar o problema.

\section{Atacando o Problema das Celas com Freqüência Muito Baixa ou Zero}

\section{a. Celas Agregadas}

Agregar celas vizinhas, omitindo variáveis ou categorias dessas variáveis, ajuda a satisfazer a condição de tamanho suficiente do subconjunto amostral correspondente à cela. Entretanto, uma abordagem desse tipo pode apresentar perda de determinadas informações, ignorando a provável contribuição de cada variável. Pode ocorrer ainda uma situação na qual determinada variável apresente apenas dois níveis, ou categorias, e a omissão de um deles acarrete perda de identidade da variável.

O teste da razão de verossimilhanças envolvendo agrupamento de celas vizinhas é baseado, de uma forma geral, na seguinte expressão:

$$
Q_{V}=-2(\mathbf{z}, \mathbf{u})_{*}^{\top}\left\{\ln (\hat{\mathbf{z}}, \hat{\mathbf{u}})_{*}-\ln (\mathbf{z}, \mathbf{u})_{*}\right\}-2(\mathbf{z}, \mathbf{u})^{\top}\{\ln (\hat{\mathbf{z}}, \hat{\mathbf{u}})-\ln (\mathbf{z}, \mathbf{u})\}
$$

em que $(\mathbf{z}, \mathbf{u})_{*}$ e $(\hat{\mathbf{z}}, \hat{\mathbf{u}})_{*}$ correspondem aos subvetores coluna das freqüências agrupadas para os valores observados e esperados respectivamente.

\section{b. Alternativas Assintóticas}

Com o objetivo de se utilizarem todas as observações disponíveis, foram propostas aproximações para a distribuição de $Q_{V}$. Koehler e Larntz (1980) mostraram que o teste baseado em $Q_{V}$ possui uma distribuição muito próxima da normal para tabelas 
esparsas. Entretanto, o uso de tais procedimentos exige certas limitações assintóticas tais como:

i) a probabilidade associada a cada cela se tornar menor quando o tamanho da amostra aumenta;

ii) nenhuma das probabilidades convergir para zero mais rapidamente do que a taxa de aumento do tamanho da amostra.

Tais condições raramente são satisfeitas.

\section{c. Adição de Constantes nas Freqüências Observadas das Celas}

Um outro procedimento freqüentemente sugerido é a adição de constantes nas celas com freqüência zero e/ou muito pequenas. Vamos explicar esse procedimento a partir de um enfoque bayesiano. Com base na expressão 3.2 (Capítulo 3) temos

$$
p(\mathbf{z}, \mathbf{u} \mid \theta, \lambda) \propto \prod_{i j k}\left(\mu_{i j k} \theta_{i j k}\right)^{z_{i j k}} \prod_{i j}\left(\lambda_{i j}^{12} \theta_{i j+}\right)^{u_{i j}^{12}} \prod_{i k}\left(\lambda_{i k}^{13} \theta_{i+k}\right)^{u_{i k}^{13}} \prod_{i}\left(\lambda_{i}^{1} \theta_{i++}\right)^{u_{i}^{1}} .
$$

Supondo que a probabilidade a priori de $(\lambda, \theta)$ siga uma distribuição de Dirichlet da forma

$$
p(\boldsymbol{\lambda}, \boldsymbol{\theta} \mid \alpha) \propto \prod_{i j k}\left(\mu_{i j k} \theta_{i j k}\right)^{\alpha-1} \prod_{i j}\left(\lambda_{i j}^{12} \theta_{i j+}\right)^{\alpha-1} \prod_{i k}\left(\lambda_{i k}^{13} \theta_{i+k}\right)^{\alpha-1} \prod_{i}\left(\lambda_{i}^{1} \theta_{i++}\right)^{\alpha-1},
$$

em que $\alpha>0$, a distribuição a posteriori de $(\lambda, \theta)$, dada pela distribuição conjunta da multinomial e da priori, também segue uma distribuição de Dirichlet da forma

$$
\begin{aligned}
p(\lambda, \boldsymbol{\theta} \mid \mathbf{z}, \mathbf{u}, \alpha) \propto & \prod_{i j k}\left(\mu_{i j k} \theta_{i j k}\right)^{z_{i j k}+\alpha-1} \prod_{i j}\left(\lambda_{i j}^{12} \theta_{i j+}\right)^{u_{i j}^{12}+\alpha-1} \prod_{i k}\left(\lambda_{i k}^{13} \theta_{i+k}\right)^{u_{i k}^{13}+\alpha-1} \\
& \prod_{i}\left(\lambda_{i}^{1} \theta_{i++}\right)^{u_{i}^{1}+\alpha-1} .
\end{aligned}
$$

As constantes adicionadas $\alpha$ correspondem a constantes de ajuste para as freqüências zero e a sua escolha se dá de forma empírica. Aliás, esse é o ponto fraco desse procedimento pois, uma escolha inapropriada de $\alpha$ pode acarretar sério viés na estimação dos parâmetros. 
A título de ilustração apresentamos na Tabela D.1 algumas escolhas de $\alpha$ feitas por alguns autores.

Tabela D.1: Valores de $\alpha$

\begin{tabular}{|c|c|c|c|}
\hline \multicolumn{4}{|c|}{ Escolha empírica do valor de $\alpha$} \\
\hline Autor & Lidstone(1920) & Perks(1947) & Jeffreys(1961) \\
\hline$\alpha$ & 1 & $1 / 2$ & $1 / c$ \\
\hline
\end{tabular}

$c$ : número de celas

Essas constantes $\alpha$ podem ser iguais para qualquer cela (o caso específico aqui) ou vinculadas a determinados parâmetros gerados pelos dados.

\section{d. Teste de Ajuste Via Simulação}

Este procedimento usa o conceito bayesiano anteriormente citado gerando uma amostra aleatória dos parâmetros da distribuição de Dirichlet a posteriori.

Denotemos o modelo saturado por $M_{1}$ que se ajusta perfeitamente ao dados. Estamos interessados em ajustar um modelo reduzido $M_{2}$. Os valores estimados para as freqüências esperadas sob o modelo $M_{2}$ são obtidos a partir da adição de $\alpha$ às freqüências observadas, sendo $\alpha$ um valor constante para todas as celas. Assim, são adotados novos valores para os vetores $\mathbf{z}$ e $\mathbf{u}$ os quais agora não possuem freqüências zero. A partir da iteração entre as expressões (3.3) e (3.4) do Capítulo 3 (passos EM), obtêm-se as estimativas das freqüências esperadas sob o modelo $M_{2}$. A simulação segue os seguintes passos:

\section{Passo 1:}

Gera-se uma amostra a partir de uma distribuição de Dirichlet a posteriori da forma

$$
p(\lambda, \boldsymbol{\theta} \mid \mathbf{z}, \mathbf{u}, \alpha) \propto \prod_{i j k}\left(\mu_{i j k} \theta_{i j k}\right)^{z_{i j k}+\alpha-1} \prod_{i j}\left(\lambda_{i j}^{12} \theta_{i j+}\right)^{u_{i j}^{12}+\alpha-1} \prod_{i k}\left(\lambda_{i k}^{13} \theta_{i+k}\right)^{u_{i k}^{13}+\alpha-1}
$$




$$
\prod_{i}\left(\lambda_{i}^{1} \theta_{i++}\right)^{u_{i}^{1}+\alpha-1}
$$

Para tal propósito, é gerada uma sucessão de números aleatórios, $\left(x_{1}, \ldots, x_{c}\right)$, seguindo distribuições gama de parâmetros $\left\{\left[z_{i j k}+\alpha\right], 1\right\},\left\{\left[u_{i j}^{12}+\alpha\right], 1\right\},\left\{\left[u_{i k}^{13}+\alpha\right], 1\right\}$ e $\left\{\left[u_{i}^{1}+\alpha\right], 1\right\}$.

Tomando-se

$$
v_{d}=\frac{x_{d}}{\sum_{d} x_{d}}, d=1, \ldots, c
$$

podemos obter o vetor $\mathbf{v}=\left(v_{1}, \ldots, v_{c}\right)$ que segue a distribuição de Dirichlet pretendida (Soares e Paulino, 2001). Na verdade, $(N+c \alpha) \mathrm{v}$ corresponde ao vetor de pseudo-freqüências observadas, que serão testadas frente ao modelo $M_{2}$ vinculado a essas pseudo-freqüências.

\section{Passo 2:}

Obtêm-se os estimadores de $\mathrm{v}$, sob a restrição imposta pelo modelo $M_{2}$, via algoritmo EM (expressões (3.3) e (3.4)).

\section{Passo 3:}

Dados os valores das freqüências pseudo-observadas $(N+c \alpha)$ v e seus estimadores sob o modelo $M_{2},(N+c \alpha) \hat{\mathbf{v}}$, calcula-se o valor da estatística de Pearson ou da RV para avaliar o ajuste do modelo. A seguir, compara-se esse valor do teste com o teste entre $M_{1}$, composto pelas freqüências verdadeiramente observadas e os respectivos valores esperados sob $M_{2}$.

\section{Passo 4:}

Repete-se os passos 1 a $3, S$ vezes. 


\section{Passo 5:}

Calcula-se o p-valor pela razão entre o número de vezes em que os valores da estatística de teste do passo 3 foram maiores que o valor da estatística de teste para ajuste do modelo $M_{2}$ frente ao $M_{1}$ com base nos dados reais e o número total de simulações $S$.

Assim, por exemplo, se $K$ valores do passo 3 foram maiores então o p-valor $($ simulado $)=\mathrm{K} / \mathrm{S}$. 


\section{Referências Bibliográficas}

[1] Agresti, A. (1990). Categorical Data Analysis. New York: John Wiley.

[2] Agresti, A. (1996). An Introduction to Categorical Data Analysis. New York: John Wiley.

[3] Baker, S. G. and Laird, N. M. (1988). Regression Analysis for Categorical Variables With Outcome Subject to Nonignorable Nonresponse. Journal of the American Statistical Association, 83, 62-69.

[4] Baker, S.G. (1996). The Analysis of Categorical Case-Control Data Subject to Nonignorable Nonresponse. Biometrics, 52, 362-369.

[5] Bishop, Y. M. M., Fienberg, S. E. and Holland, P. W. (1975). Discrete Multivariate Analysis. Cambrige, MA: The MIT Press.

[6] Clogg, C. C., Rubin, D. B.. Schenker, N. and Schultz, B. (1991). Multiple Imputation of Industry and Occupation Codes in Census Public-Use Samples Using Bayesian Logistic Regression. Journal of the American Statistical Association, $86,68-78$.

[7] Cochran, W. G. (1942). The $\chi^{2}$ Correction for Continuity. Iowa State Journal of Science, 16, 421-436.

[8] Cochran, W. G. (1954). Some Methods for Strengthening the Common $\chi^{2}$ Tests. Biometrics,10, 417-451.

[9] Cramér, H. (1946). Mathematical Methods of Statistics. Princeton University Press.

[10] Datafolha. Página www.datafolha.com.br. Eleições para presidente, 2002. 
[11] Deming, W. E. and Stephan, F. F. (1940). On a least Squares Adjustment of a Sampled Frequency Table When the Expected Marginals Totals are Known. Annals of Mathematical Statistics, 11, 427-444.

[12] Dempster, A. P., Laird, N. M., and Rubin, D. B. (1977). Maximum likelihood from incomplete data via the EM algorithm (with discussion). Journal of the Royal Statistical Society B, 39,1-38.

[13] Dudewicz, E. J. and Mishra, S. N. (1988). Modern Mathematical Statistics. New York: John Wiley \& Sons.

[14] Fay, R. E. (1986). Causal Models for Patterns of Nonresponse. Journal of the American Statistical Association, 77, 270-278.

[15] Fienberg, S. E. (1980). The Analysis Of Cross-Classified Categorical Data. Cambridge: The MIT Press.

[16] Fisher, R. A. and Yates, F.(1938). Statistical Tables. Edinburgh: Oliver and Boyd.

[17] Jeffreys, J. (1961). Theory of Probabilities.Oxford: Clarendon Press.

[18] Koehler, K. and Larntz, K. (1980). An empirical investigation of goodness-of-fit statistics for sparse multinomials. Journal of the American Statistical Association, 75, 336-344.

[19] Koehler, K. (1986). Goodness-of-fit tests for log-linear models in sparse contingency tables. Journal of the American Statistical Association, 81, 483-493.

[20] Larntz, K. (1978). Small-sample comparison of exact levels for chi-squared goodness-of-fit statistics. Journal of the American Statistical Association, 73, 253-263.

[21] Lidstone, G. J. (1920). Note on the general case of the Bayes-Laplace Formula for inductive or a posteriori probabilities. Transactions of the Faculty of Actuaries, $8,182-192$.

[22] Little, R.J.A., and Rubin, D. B. (1987). Statistical Analysis With Missing Data. New York: John Wiley. 
[23] McLachlan, G. J., and Krishnan, T. (1997). The EM Algorithm and Extensions. New York: John Wiley \& Sons.

[24] Park, T. and Brown, M. B. (1994). Models for Categorical Data With Nonignorable Nonresponse. Journal of the American Statistical Association, 89, 44-52.

[25] Perks, W. (1947). Some Observations on Inverse Probability including a New Indifference Rule. Journal of the Institute of Actuaries, 73, 285-334.

[26] Soares, P. and Paulino, C. D. (2001). Incomplete Categorical Data Analysis: A Bayesian Perspective. Journal of Statistical Computation and Simulation, 69, 157-170.

[27] Stempliuk, V. (2002). Pesquisa sobre Consumo de Drogas na Universidade de São Paulo. Tese de Doutorado. Instituto de Psiquiatria. USP. A ser defendida.

[28] Williamson, G. D. and Haber, M. (1994). Models for three-dimensional contingency tables. with completely and partially cross-classified data. Biometrics, 50,194-203.

[29] Yarnold, J. K. (1970).The Minimum Expectation in $\chi^{2}$ Goodness-of-Fit Tests and Accuracy of Approximations for the Null Distribution. Journal of the American Statistical Association, 65, 864-886. 